\title{
Catalyst-Free, Metal-Free, and Chemoselective Transamidation of Activated Secondary Amides
}

\author{
Rajagopal Ramkumar \\ Srinivasan Chandrasekaran* (D) \\ Department of Organic Chemistry, Indian Institute of Science, \\ Bangalore 560012, India \\ scn@iisc.ac.in
}

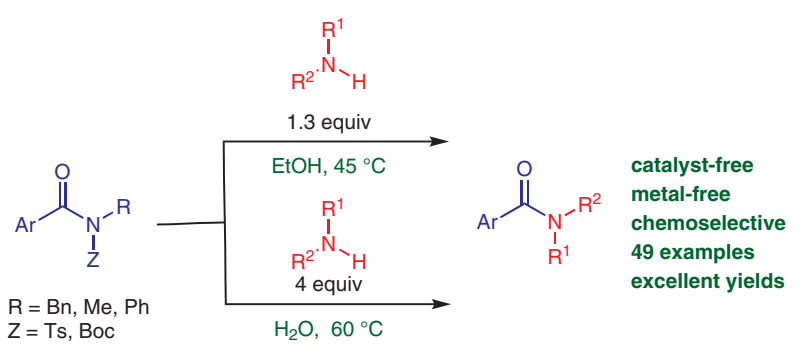

Received: 04.09.2018

Accepted after revision: 21.09.2018

Published online: 18.10 .2018

DOI: 10.1055/s-0037-1610664; Art ID: ss-2018-z0592-op

Abstract A simple protocol, which is catalyst-free, metal-free, and chemoselective, for transamidation of activated secondary amides in ethanol as solvent under mild conditions is reported. A wide range of amines, amino acids, amino alcohols, and the substituents, which are problematic in catalyzed transamidation, are tolerated in this methodology. The transamidation reaction was successfully extended to water as the medium as well. The present methodology appears to be better than the other catalyzed transamidations reported recently.

Keywords amino acid, catalyst-free, metal-free, chemoselective, secondary amide, transamidation

The interconversion of one amide to another amide is known as the transamidation reaction. The transamidation reactions are one of the most fundamental transformations in synthetic organic chemistry because amides are ubiquitous in nature and are most important functional molecules in industry. ${ }^{1}$ However, the notorious stability ${ }^{2}$ of the amide group is a major challenge arising out of the high kinetic barrier, the thermodynamic factor to break the $\mathrm{C}-\mathrm{N}$ bond, and the resonance stabilization of the amide group. ${ }^{3}$ In spite of this, notable headway has been made with transamidation of primary amides. ${ }^{4}$ Recently several successful attempts have been reported for transamidation reactions of secondary amides. ${ }^{5}$ The Lewis acid catalyzed transamidation reaction of secondary amide is accomplished by the activation of the amide carbonyl group. ${ }^{6}$ Gellman and coworkers reported the use of dimeric aluminum complex ${ }^{6 a}$ and Bertrand reported the use of excess $\mathrm{AlCl}_{3}$ for simple substrates. ${ }^{6 b}$ Garg and co-workers achieved transamidation of Boc-activated aliphatic and aryl substituted secondary amides in a two-step process using nickel catalysis (Scheme 1a). ${ }^{7}$ More recently, a metal-free transamidation involving triethylamine $^{8 \mathrm{a}, \mathrm{b}}$ and a Pd-NHC complex-mediated ${ }^{8 \mathrm{c}}$ transamidation of secondary amides were reported by Szostak (Scheme 1b). ${ }^{8}$ Despite these reports, a more general approach to the problem of transamidation has remained a challenge and a more sustainable green methodology is warranted. Herein, we describe the results of a successful approach and report a highly selective methodology for transamidation of secondary amides under catalyst-free conditions in ethanol or water as solvent (Scheme 1c). The $\mathrm{N}$-functionalized substrates with appropriate activating groups such as Boc or Ts or aryl sulfonyl groups generally undergo an efficient transamidation reaction smoothly (Scheme 1c). ${ }^{9}$

$$
\text { a) } \mathrm{Ni} \text {-catalyzed transamidation of secondary amides (previous work) }
$$

b) Metal-free transamidation of secondary amides (previous work) ${ }^{8 \mathrm{a}}$

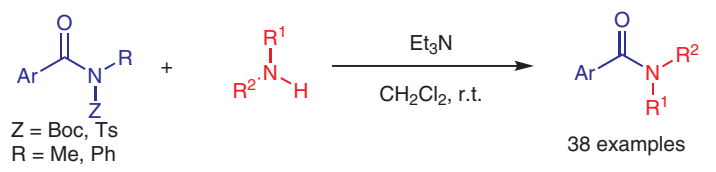

C) Catalyst-free and metal-free transamidation of secondary amides (This work)

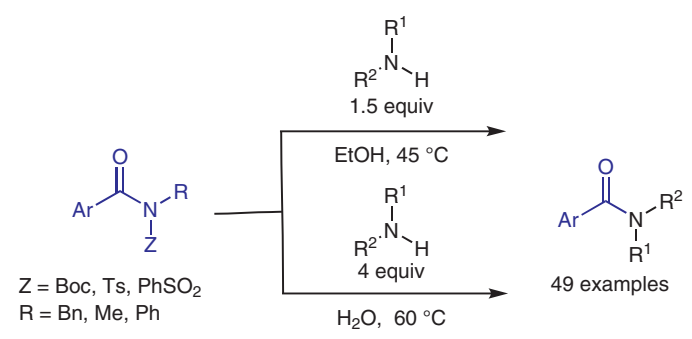

Scheme 1 Transamidation of secondary amides 
Initially, when the transamidation was performed with activated secondary benzamide $\mathbf{1 a}$ (1 equiv) and piperidine (2a; 1.3 equiv) at room temperature there was no reaction in ethanol or dichloromethane (Table 1 , entries a, b) even after 24 hours. The above reaction performed at $45{ }^{\circ} \mathrm{C}$ in dichloromethane gave the transamidation product 3a in $68 \%$ yield after 12 hours (entry c). Encouraged by this initial result, the reaction was tested in other solvents like toluene and THF at higher temperatures $\left(45-80^{\circ} \mathrm{C}\right)$ and the product 3a was obtained in moderate yields (entries d, e). Interestingly, the transamidation reaction was found to occur when performed in water at $60^{\circ} \mathrm{C}$, affording the secondary amide 3a in $58 \%$ yield (entry f). Finally, when the transamidation reaction was performed in ethanol at $45^{\circ} \mathrm{C}$ it gave an excellent yield (99\%) of 3a in 1.5 hours (entry g). It is pertinent to point out that the reaction of unactivated amides like $\mathrm{N}$ benzyl- $\mathrm{N}$-methylbenzamide with piperidine under the same reaction conditions does not provide any transamidation product.

Table 1 Optimization of Reaction Conditions ${ }^{\mathrm{a}}$

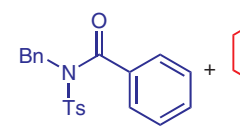

$1 \mathrm{a}$

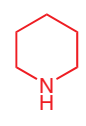

2a

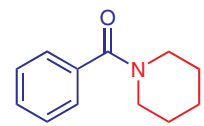

3a

\begin{tabular}{ccccc}
\hline Entry & Solvent & Temp $\left({ }^{\circ} \mathrm{C}\right)$ & Time $(\mathrm{h})$ & Yield $(\%)^{\mathrm{b}}$ \\
\hline $\mathrm{a}$ & $\mathrm{EtOH}$ & r.t. & 24 & 0 \\
$\mathrm{~b}$ & $\mathrm{CH}_{2} \mathrm{Cl}_{2}$ & r.t. & 24 & 0 \\
$\mathrm{c}$ & $\mathrm{CH}_{2} \mathrm{Cl}_{2}$ & 45 & 12 & 68 \\
d & toluene & 45 & 12 & 61 \\
e & $\mathrm{THF}$ & 80 & 24 & 59 \\
$\mathrm{f}$ & $\mathrm{H} 2 \mathrm{O}$ & 60 & 12 & $58^{\mathrm{c}}$ \\
$\mathrm{g}$ & $\mathbf{E t O H}$ & $\mathbf{4 5}$ & $\mathbf{1 . 5}$ & $\mathbf{9 9}$
\end{tabular}

a Reaction conditions: activated secondary amide substrate 1 a (1 equiv), amine (1.3 equiv), EtOH ( $1 \mathrm{~mL}$ ).

b Isolated yields.

' Reaction was performed with activated secondary amide substrate 1a (1 equiv) and amine (1.3 equiv) in $\mathrm{H}_{2} \mathrm{O}(1 \mathrm{~mL})$.

With optimized conditions in hand, the scope and generality of the transamidation protocol were explored with a variety of primary and secondary amine partners with different activated amides 1 (Figure 1, Scheme 2). The cyclic secondary amines such as pyrrolidine and morpholine could be utilized for the transamidation reaction with $\mathbf{1 a}$ to furnish the corresponding products $\mathbf{3 b}$ and $\mathbf{3 c}$, respectively, in excellent yields (97\% and 99\%). Substrates with electrondonating substituents on the aryl ring of activated secondary amides $\mathbf{1 b}, \mathbf{c}$ readily undergo transamidation to give products $\mathbf{3 d}, \mathbf{e}$ in excellent yields. Interestingly, this green protocol tolerates several functional groups such as bromo and iodo that would be problematic substrates in the metal- catalyzed transamidation to form $\mathbf{3 f}-\mathbf{i}^{8}{ }^{8,10}$ Further, the N-activated heterocyclic secondary amides $\mathbf{1 f}-\mathbf{h}$ also furnished the corresponding transamidation products $\mathbf{3} \mathbf{j}-\mathbf{l}$ in excellent yields. The reaction of secondary amide with $\mathrm{N}$-methylaniline also gave the transamidation product $\mathbf{3 m}$ in $95 \%$ yield.

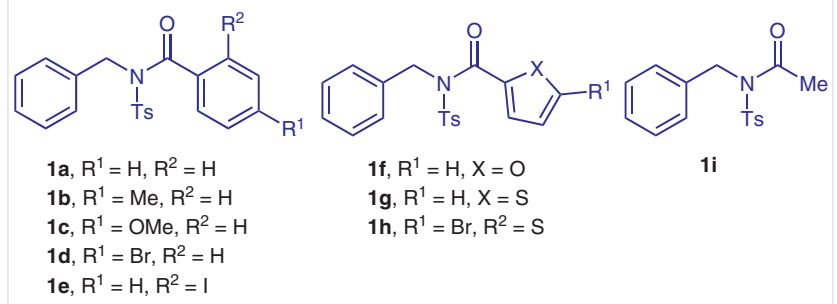

Figure 1 Activated amides used in this work

Further, we examined the scope of reaction with different anilines as shown by the formation of secondary amides 3n-p. In all the cases, the reactions occur very smoothly without affecting the yield. ${ }^{8}$ Reaction of benzylamine with activated amide 1a also furnished the secondary amide 3q in high yield. Additionally, propargylamine could also be used to form the secondary amide $3 \mathbf{r}$ in $97 \%$ yield. Cyclohexylamine or hindered 1-adamantylamine also undergo smooth transamidation with 1a to furnish the corresponding secondary amides 3s,t in $98 \%$ and $94 \%$ yield, respectively. The results of this study are summarized in Scheme 2.

The scope of this methodology was then extended to tosyl-activated $\mathrm{N}$-methylamide derivatives $\mathbf{4}$ with piperidine and $\alpha$-branched amines. The amide substrates $\mathbf{4 a , b}$ containing either electron-donating substituents or electron-withdrawing substituents on the aryl ring furnish the corresponding amides $\mathbf{5 a}, \mathbf{b}$ in excellent yields. $\alpha$-Branched amine such as isopropylamine and primary amine like $n$ butylamine also react with amide $\mathbf{4 c}$ leading to the formation of the desired secondary amides $\mathbf{5 c , d}$ in high yields (Scheme 3).

It was of interest to study the reaction of amides activated with N-Boc group in the transamidation reaction. Reaction of N-Boc-activated amides $\mathbf{6 a}, \mathbf{b}$ with secondary or primary amines was explored in ethanol at $45{ }^{\circ} \mathrm{C}$ and the results are presented in the Scheme 4 . The reactions generally yielded the expected products $\mathbf{7 a - f}$ in almost quantitative yield.

To evaluate the efficiency of this protocol, the transamidation of activated secondary amide substrate 1a was performed with amino acid and amino alcohol derived nucleophiles. All the nucleophiles derived from amino acids, phenylalanine, alanine, and methionine, and the amino alcohols could be utilized for the reaction, leading to the corresponding secondary amides 8a-d, respectively in high yields (Scheme 5). It is of interest to note that the reaction is chemoselective in that only $\mathrm{N}$-nucleophiles react in the 


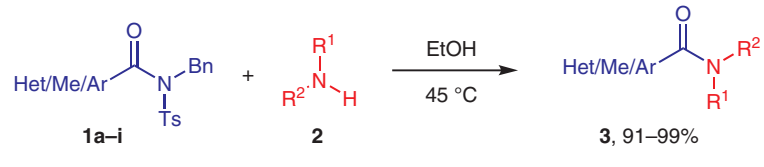<smiles>O=C(c1ccccc1)N1CCCCC1</smiles>

3a, $99 \%$<smiles>O=C(c1ccc(Br)cc1)N1CCOCC1</smiles>

3g, $91 \%$<smiles>O=C(c1ccccc1)N(c1ccccc1)c1ccccc1</smiles>

3m, 95\%<smiles>O=C(c1ccccc1)N1CCOCC1</smiles>

3b, $97 \%$<smiles>O=C(c1ccccc1I)N1CCCCC1</smiles>

3h, $93 \%$<smiles>O=C(Nc1ccccc1)c1ccccc1</smiles>

3n, $96 \%$<smiles>O=C(NC1CCCCC1)c1ccccc1</smiles>

3 s, $98 \%$<smiles>O=C(c1ccccc1)N1CCCC1</smiles>

3c, $99 \%$<smiles>O=C(c1ccccc1I)N1CCOCC1</smiles>

3i, $91 \%$<smiles>O=C(Nc1ccc(I)cc1)c1ccccc1</smiles>

3o, $92 \%$<smiles>O=C(NC12CC3CC(CC(C3)C1)C2)c1ccccc1</smiles>

3t, $94 \%$<smiles>Cc1ccc(C(=O)N2CCCCC2)cc1</smiles>

3d, $96 \%$<smiles>O=C(c1ccco1)N1CCCCC1</smiles>

3j, $97 \%$<smiles>O=C(Nc1cc(Cl)cc(Cl)c1)c1ccccc1</smiles>

3p, $90 \%$<smiles>CCCCNC(=O)c1ccccc1</smiles><smiles>CCCCNC(C)=O</smiles>

3u, $97 \%$<smiles>COc1ccc(C(=O)N2CCOCC2)cc1</smiles>

3e, $95 \%$<smiles>O=C(c1ccc(Br)o1)N1CCCCC1</smiles>

3k, $96 \%$<smiles>O=C(NCc1ccccc1)c1ccccc1</smiles>

3q, $98 \%$<smiles>O=C(c1ccc(Br)cc1)N1CCCCC1</smiles>

3f, $94 \%$<smiles>O=C(c1cccs1)N1CCCCC1</smiles>

3I, $96 \%$<smiles>C#CCNC(=O)c1ccccc1</smiles>

3r, $97 \%$

Scheme $\mathbf{2}$ Transamidation of tosyl-activated $\mathrm{N}$-benzylamide $\mathbf{1}$ with various amines $\mathbf{2}$. Reagents and conditions: activated secondary amide substrate $\mathbf{1 a}$ (1 equiv), amine (1.3 equiv), EtOH ( $1 \mathrm{~mL})$. Isolated yields are shown.

presence of $\mathrm{O}$-nucleophiles in amino alcohols. This selectiv-

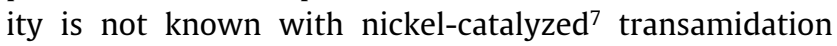
reactions.

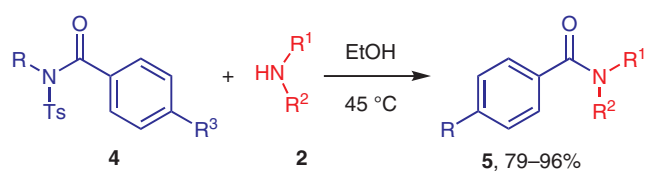

4a, $\mathrm{R}=\mathrm{Me}, \mathrm{R}^{3}=\mathrm{OMe}$

$4 \mathrm{~b}, \mathrm{R}=\mathrm{Me}, \mathrm{R}^{3}=\mathrm{NO}_{2}$

$4 c, R=M e, R^{3}=H$

$4 d, R=P h, R^{3}=H$

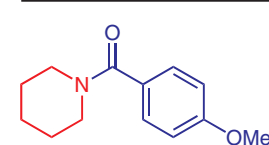

5a, $96 \%$<smiles>CCCCNC(=O)c1ccccc1</smiles>

5d, $98 \%$<smiles>O=C(c1ccc([N+](=O)[O-])cc1)N1CCCCC1</smiles>

$5 b, 89 \%$<smiles>CC(C)(C)NC(=O)c1ccccc1</smiles>

5e, $79 \%$

Scheme 3 Transamidation of tosyl-activated $\mathrm{N}$-methylamide $\mathbf{4}$ with amines 2 . Reagents and conditions: activated secondary amide substrate 5 (1 equiv), amine (1.3 equiv), EtOH (1 mL). Isolated yields are shown.

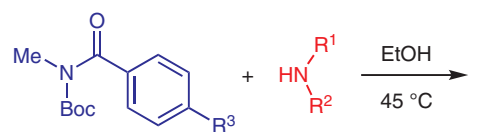

$$
\text { 6a, } R^{3}=O M e
$$

$6 b, \mathrm{R}^{3}=\mathrm{CF}_{3}$

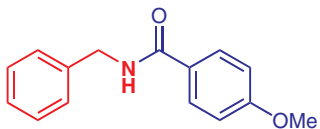

7a, $95 \%$<smiles>CC(C)NC(=O)c1ccccc1</smiles>

5c, $94 \%$<smiles>O=C(NC1CCCCC1)c1ccc(C(F)(F)F)cc1</smiles>

7c, $95 \%$<smiles>O=C(c1ccc(C(F)(F)F)cc1)N1CCOCC1</smiles><smiles>O=C(c1ccc(C(F)(F)F)cc1)N1CCCC1</smiles>

7f, $97 \%$

Scheme 4 Transamidation of Boc-activated $\mathrm{N}$-methylamide $\mathbf{6}$ with amines 2. Reagents and conditions: Boc-activated secondary amide 7 (1 equiv), amine (1.3 equiv), EtOH ( $1 \mathrm{~mL}$ ). Isolated yields are shown. 
<smiles>CN(Cc1ccccc1)C(=O)c1ccccc1</smiles>

1a
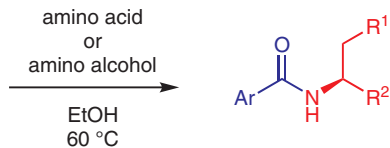

8, $89-97 \%$<smiles>COC(=O)[C@H](Cc1ccccc1)NC(=O)c1ccccc1</smiles>

8 a, $97 \%$<smiles>CCOC(=O)[C@H](C)NC(=O)c1ccccc1</smiles>

$8 c, 94 \%$<smiles>CCC[C@H](NC(=O)c1ccccc1)C(=O)OC</smiles>

$8 b, 94 \%$<smiles>CCC(C)[C@H](CO)NC(=O)c1ccccc1</smiles>

$8 d, 89 \%$

Scheme 5 Transamidation of Ts-activated $\mathrm{N}$-benzylamide $\mathbf{1}$ a with amino acids and amino alcohols. Reagents and conditions: amide $1 \mathbf{a}$ ( 1 equiv), amino acid, or amino alcohol (1.5 equiv), EtOH $(1 \mathrm{~mL})$ at $60{ }^{\circ} \mathrm{C}$. Isolated yields are shown.

At this stage, we decided to test the feasibility of this transamidation in water. Our initial studies with 1a and piperidine ( 1.5 equiv) in water gave only $58 \%$ the product 3a. When the stoichiometry of the amine was increased to 4 equivalents at $60{ }^{\circ} \mathrm{C}$, the yield of the product 3a went up to to $97 \%$. Some of the successful experiments in water are presented in Scheme 6.

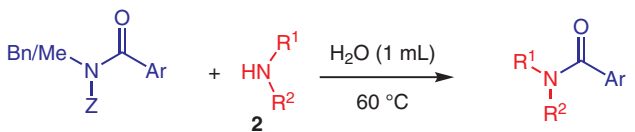

$$
\begin{aligned}
& \text { 1a NBn } Z=\text { Ts } \longrightarrow \text { 3a, 3b, 3n } \\
& \text { 1b NBn } Z=T s \quad \longrightarrow 3 d \\
& \text { 1f } \mathrm{NBn} \quad \mathrm{Z}=\mathrm{Ts} \longrightarrow 9 \\
& \text { 6b NMe } \quad Z=\mathrm{Boc} \longrightarrow 7 \mathrm{~b}
\end{aligned}
$$<smiles>O=C(c1ccccc1)N1CCCCC1</smiles>

3a, $97 \%$<smiles>O=C(c1ccccc1)N1CCOCC1</smiles><smiles>O=C(c1ccoc1)N1CCOCC1</smiles>

9, $92 \%$ 3b, $95 \%$

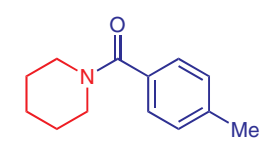

3d, $94 \%$<smiles>O=C(Nc1ccccc1)c1ccccc1</smiles>

3n, $89 \%$

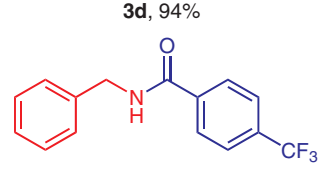

$7 b, 98 \%{ }^{c}$
Scheme 6 Transamidation of activated secondary amides in water. Reagents and conditions: Amide $\mathbf{1 a - b}, \mathbf{f}$ (1 equiv), amine $\mathbf{2}$ (4 equiv), $\mathrm{H}_{2} \mathrm{O}$ ( $1 \mathrm{~mL}$ ) at $60^{\circ} \mathrm{C}$; amide $\mathbf{6 b}$ (1 equiv), amine $\mathbf{2}$ (4 equiv), $\mathrm{H}_{2} \mathrm{O}(1 \mathrm{~mL})$ at $60{ }^{\circ} \mathrm{C}$. Isolated yields are shown.

Having successfully carried out many transamidation reactions under catalyst-free, metal-free conditions, we decided to compare our results with similar transamidation reaction reported in the literature recently under nickelcatalyzed $^{7}$ condition as well the reaction performed in the presence of triethylamine. ${ }^{8}$

The Boc-activated amide 11a on transamidation with sterically hindered 1-adamantylamine and 2,6-dimethylaniline in $\mathrm{Et}_{3} \mathrm{~N}$ is reported to give no product while under $\mathrm{Ni}$-catalyzed condition ${ }^{7}$ gave $58 \%$ and $49 \%$ of corresponding amides (3t and 12a). Under our reaction condition both the amines reacted with 11a to give $73 \%$ and $69 \%$ yield of products (Table 2 , entries 1,2 ). While the reaction of benzylamine and morpholine with $\mathbf{1 1 b}$ has not been reported under nickel catalysis, ${ }^{7}$ under $\mathrm{Et}_{3} \mathrm{~N}$ catalysis benzylamine afforded excellent yield of the product 12b (Table 2, entries $3,4) .^{8}$ In the present protocol, both the amines react with $\mathbf{1 1 b}$ to give higher yield of the transamidation products $\mathbf{3 g}$ and $\mathbf{1 2 b}$.

Verho reported ${ }^{11}$ a protocol where they find that the reaction of $\mathbf{1 0}$ with $(R)$-1-methylbenzylamine (5 equiv) gave the corresponding amide in 52\% yield after 24 hours. However, using our methodology the same reaction of $\mathbf{1 0}$ with chiral amine $(R)$-1-methylbenzylamine (1.5 equiv) gave the product 12c without loss of optical purity in $88 \%$ yield in 6 hours (Table 2, entry 5). Thus our methodology appears to be as good as the other reported transamidations in the literature and in some cases better.

\section{Mechanistic Studies}

A series of competitive experiments were performed using $\mathrm{N}$-activated amide 1a with various amine nucleophiles to identify the selectivity patterns of the transamidation (Scheme 7). To study the effect of ring size, we compared the reaction of cyclic amines piperidine and pyrrolidine with 1a. The major product obtained was $\mathbf{3 c}$ (reaction with pyrrolidine) in $66 \%$ yield while $3 \mathbf{a}$ was found to be the minor product.

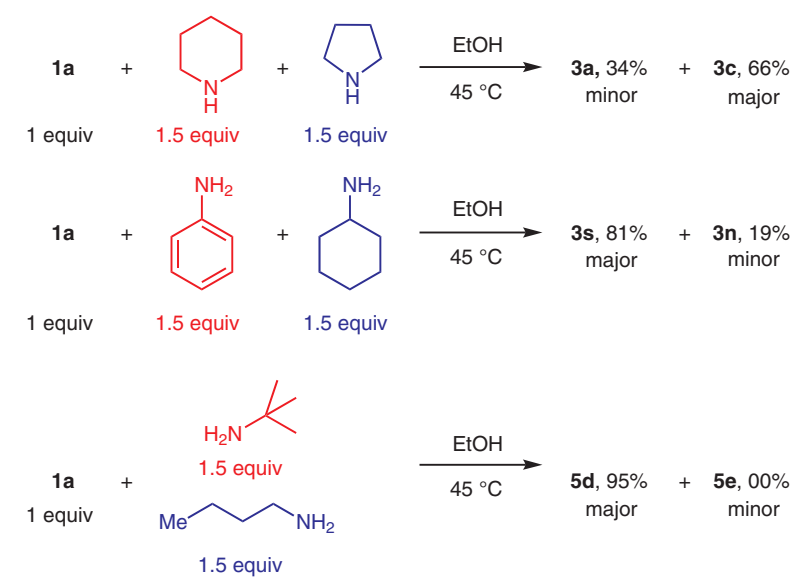

Scheme 7 Competitive reactivity of various amines

The reaction of aryl- versus alkylamine was tested by the reaction of aniline and cyclohexylamine with $\mathbf{1 a}$. The product 3s derived from cyclohexylamine was found to be 
Table 2 Comparison Table of Transamidation of $\mathrm{N}$-Activated $2^{\circ}$ Amides Using [Ni] Catalysis, Metal-Free Conditions and Our Protocol ${ }^{\mathrm{a}}$

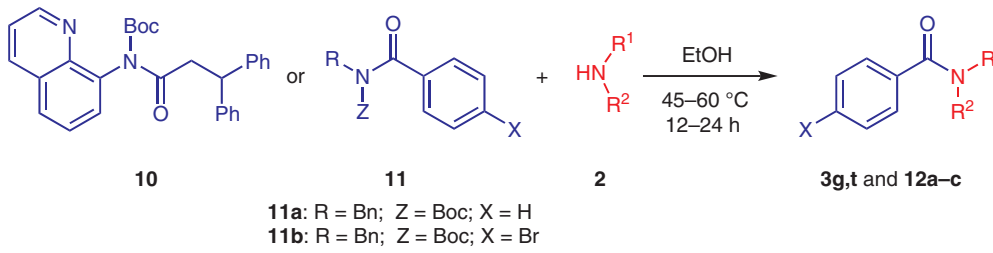

\begin{tabular}{|c|c|c|c|c|c|c|c|}
\hline Entry & Amide & Amine & Product & Ni catalyst $^{7}$ & $\mathrm{Et}_{3} \mathrm{~N}^{8}$ & Metal-free $^{11}$ & Our work \\
\hline 1 & $11 a$ & 1-adamantylamine & $3 t$ & 58 & no reaction & not reported & 73 \\
\hline 2 & $11 a$ & 2,6-dimethylaniline & $12 a$ & 49 & no reaction & not reported & 69 \\
\hline 3 & $11 b$ & benzylamine & $12 b$ & not reported & 98 & not reported & 96 \\
\hline 4 & $11 b$ & morpholine & $3 g$ & not reported & not reported & not reported & 78 \\
\hline 5 & 10 & $(R)$-1-methylbenzylamine ${ }^{c}$ & $12 \mathrm{c}$ & not reported & not reported & 52 & 88 \\
\hline
\end{tabular}

a Reaction conditions: Amide 11 (1 equiv), amine 2 (1.3 equiv), EtOH (1 mL) at $45-60{ }^{\circ} \mathrm{C}$.

b Isolated yields.

c Amide 10 (1 equiv), amine 2 ( 1.5 equiv), $\mathrm{EtOH}(1 \mathrm{~mL})$ at $60{ }^{\circ} \mathrm{C}, 6 \mathrm{~h}$.

the major product (81\%). The involvement of the steric factors in the reaction was tested using $n$-butylamine and tertbutylamine as partners for transamidation. The only product obtained in this case was the amide $\mathbf{5 d}$ resulting from the reaction of $n$-butylamine.

The study of selectivity and reactivity of Ts- and Boc-activated amides containing electron-donating or electronwithdrawing substituents with piperidine was carried out (Scheme 8). When a 1:1 mixture of Ts-activated amides 4a and $\mathbf{4 b}$ was treated with piperidine $\left(\mathrm{EtOH}, 45^{\circ} \mathrm{C}, 1.5 \mathrm{~h}\right)$ the major product was found to be $\mathbf{5 a}$. Similarly, the reaction of a 1:1 mixture of Boc-activated amides $\mathbf{6 a}$ and $\mathbf{6 b}$ with piperidine gave $\mathbf{7 d}$ as the major product. Additionally we tested the competitive reactivity of Ts-activated amide 1a and Boc-activated amide 11a with piperidine at different time intervals. The amide 11a started to react with piperidine only after 1 hour but 1a was already fully consumed. It shows that $\mathrm{N}$-Ts-activated amide $\mathbf{1}$ a reacts faster than Bocactivated amide 11a.

Based on the above observations we propose a reaction mechanism, which is similar to the one proposed by Szostak. ${ }^{8 \mathrm{a}, \mathrm{b}}$ The transamidation of secondary amides is achieved by exothermic nucleophilic addition of amine to form intermediate 13. Subsequent release of the $\mathrm{N}$-functionalized amine 14, which is less nucleophilic than the amine involved in the transamidation reaction leads to the formation of product 3 (Scheme 9 ).<smiles>COc1ccc(C(=O)N([18O])[Na])cc1</smiles><smiles>[14CH3]</smiles>

2 equiv<smiles>COc1ccc(C(=O)N(C)C)cc1</smiles>

$$
\begin{gathered}
6 \mathbf{a} \\
2 \text { equiv }
\end{gathered}
$$<smiles>O=C(c1ccccc1)N(Cc1ccccc1)Cc1ccccc1</smiles>

1 equiv<smiles>CN(C)C(=O)c1ccc([N+](=O)[O-])cc1</smiles><smiles>[131In]</smiles><smiles>CN(C(=O)O)C(=O)c1ccc(C(F)(F)F)cc1</smiles>

6b

2 equiv<smiles>O=C(c1ccccc1)N(Br)Cc1ccccc1</smiles>

1 equiv<smiles>C1CCNCC1</smiles><smiles>C#CC(C)(C)C</smiles>
$\underset{\text { major }}{\mathbf{5 a}}+\underset{\text { minor }}{\mathbf{5 b}}$

1 equiv<smiles>C1CCNCC1</smiles><smiles>C[13CH3]</smiles>
$5 a+7 d$ 1 equiv<smiles>C1CCC2CNCC2C1</smiles><smiles>CCOC(=O)NCc1ccccc1</smiles>

2 equiv

Scheme 8 Competition studies with N-Ts- and N-Boc-activated amides 


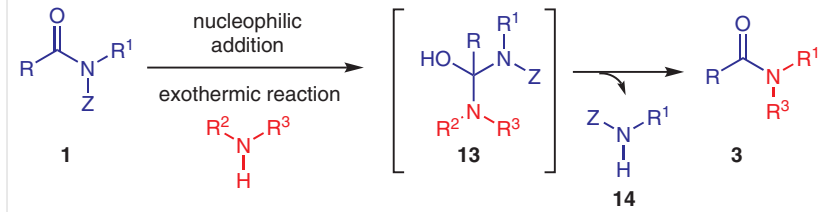

Scheme 9 Proposed Mechanism

\section{Conclusion}

In summary, we have developed a simple protocol for the transamidation of a number of activated secondary amides using a broad range of amines in ethanol as solvent under catalyst-free and metal-free conditions. We have also extended the scope of the transamidation to water medium. This effective procedure is highly chemoselective as shown in the reaction of amino acid and amino alcohols. The stereochemical integrity of the amino acid and amino alcohol derivatives is preserved in the reaction. The present methodology appears to be better than other catalyzed transamidations reported in the literature recently.

Melting points were determined using a capillary melting point apparatus and are uncorrected. All the transamidation reactions were performed using sealed vial under air atmosphere, unless stated otherwise. The starting materials were prepared based on the previously reported literature method. The solvents purchased were of the laboratory grade (LR) and used as received or purified by distillation following standard procedure. All other chemicals were purchased from Sigma-Aldrich, Alfa Acer, and TCI. ${ }^{1} \mathrm{HNMR}$ spectra were recorded on a Bruker Avance at $400 \mathrm{MHz}$ using $\mathrm{CDCl}_{3}$ or DMSO- $d_{6}$ in ppm ( $\delta$ ) related to TMS $(\delta=0.00)$ as an internal standard and are reported as follows; chemical shift (ppm), multiplicity (standard abbreviations), coupling constant $(\mathrm{Hz})$, and integration. ${ }^{13} \mathrm{C}$ NMR spectra were recorded at $100 \mathrm{MHz}$ in $\mathrm{CDCl}_{3}$ or DMSO- $d_{6}$. Chemical shifts are reported in delta $(\delta)$ units, parts per million (ppm) relative to the center of the triplet at $77.7 \mathrm{ppm}$ for $\mathrm{CDCl}_{3}$ or $39.5 \mathrm{ppm}$ for DMSO- $d_{6}$. Carbon types were determined from ${ }^{13} \mathrm{C}$ NMR and DEPT experiments. Mass spectra were measured with Micromass Q-Tof (ESI-HRMS). Optical rotations were measured on JASCO P-2000 polarimeter at r.t. using $50 \mathrm{~mm}$ cell of $1 \mathrm{~mL}$ capacity. TLC was performed on silica gel GF-254 and components visualized by observation under $\mathrm{I}_{2} / \mathrm{UV}$ light at $254 \mathrm{~nm}$. Column chromatography was performed on silica gel (230-400 mesh).

\section{N-Ts-Activated Secondary Amides; General Procedure}

An oven-dried $100 \mathrm{~mL}$ round-bottomed flask was charged with $\mathrm{N}$ benzyl-4-methylbenzenesulfonamide ( $1.0 \mathrm{mmol}, 1.0$ equiv), and $\mathrm{NaH}$ (1.5 mmol, 1.5 equiv) in anhyd THF $(20 \mathrm{~mL})$. A solution of the respective acyl chloride $(1.0 \mathrm{mmol}, 1.0$ equiv) in anhyd THF $(10 \mathrm{~mL})$ was added at $0{ }^{\circ} \mathrm{C}$. The reaction mixture was stirred at r.t. $\left(25^{\circ} \mathrm{C}\right)$ for $3 \mathrm{~h}$. The reaction was monitored by TLC. After completion of the reaction, the mixture was quenched with $\mathrm{H}_{2} \mathrm{O}$ and extracted with $\mathrm{CH}_{2} \mathrm{Cl}_{2}(3 \times$ $25 \mathrm{~mL}$ ). The combined organic layers were washed with brine (20 $\mathrm{mL}$ ), dried (anhyd $\mathrm{Na}_{2} \mathrm{SO}_{4}$ ), and the filtrate was evaporated under reduced pressure. The crude product was purified by column chromatography on silica gel (EtOAc/PE) to afford the desired $N$-tosyl-activated secondary amide.
$\boldsymbol{N}$-Benzyl- $\boldsymbol{N}$-tosylbenzamide (1a) ${ }^{12}$

Eluent: PE/EtOAc (90:10); white solid; yield: $1.29 \mathrm{~g}$ (92\%); mp 95-97 ${ }^{\circ} \mathrm{C}$. ${ }^{1} \mathrm{H}$ NMR $\left(\mathrm{CDCl}_{3}, 400 \mathrm{MHz}\right): \delta=2.41\left(\mathrm{~s}, 3 \mathrm{H}, \mathrm{CH}_{3}\right), 5.01\left(\mathrm{~s}, 2 \mathrm{H}, \mathrm{CH}_{2}\right)$, 7.21-7.26 (m, 7 H, ArH), $7.34(\mathrm{t}, J=7.2 \mathrm{~Hz}, 2 \mathrm{H}, \mathrm{ArH}), 7.46$ (t, J = 7.6 Hz, $3 \mathrm{H}, \mathrm{ArH}), 7.63(\mathrm{~d}, J=8.0 \mathrm{~Hz}, 2 \mathrm{H}, \mathrm{ArH})$.

${ }^{13} \mathrm{C}$ NMR $\left(\mathrm{CDCl}_{3}, 100 \mathrm{MHz}\right): \delta=21.61,51.25,127.75,127.91,128.18$, $128.50,128.55,129.37,130.14,131.70,133.72,134.90,135.85$, $136.21,144.70,171.56$.

\section{$\boldsymbol{N}$-Benzyl-4-methyl- $\boldsymbol{N}$-tosylbenzamide (1b) $)^{12}$}

Eluent: PE/EtOAC (90:10); colorless oil; yield: $256 \mathrm{mg}$ (88\%).

${ }^{1} \mathrm{H}$ NMR $\left(\mathrm{CDCl}_{3}, 400 \mathrm{MHz}\right): \delta=2.35\left(\mathrm{~s}, 3 \mathrm{H}, \mathrm{CH}_{3}\right), 2.40\left(\mathrm{~s}, 3 \mathrm{H}, \mathrm{CH}_{3}\right)$, $4.94\left(\mathrm{~s}, 2 \mathrm{H}, \mathrm{CH}_{2}\right), 7.14$ (d, $\left.J=8.0 \mathrm{~Hz}, 2 \mathrm{H}, \mathrm{ArH}\right), 7.19-7.25(\mathrm{~m}, 7 \mathrm{H}$, $\mathrm{ArH}), 7.41(\mathrm{~d}, J=7.6 \mathrm{~Hz}, 2 \mathrm{H}, \mathrm{ArH}), 7.60(\mathrm{~d}, J=8.0 \mathrm{~Hz}, 2 \mathrm{H}, \mathrm{ArH})$.

${ }^{13} \mathrm{C} \mathrm{NMR}\left(\mathrm{CDCl}_{3}, 100 \mathrm{MHz}\right): \delta=21.56,21.59,51.32,127.68,127.93$, $128.47,128.50,128.60,128.86,129.33,132.05,135.89,136.18$, $142.61,144.54,171.65$.

\section{$\boldsymbol{N}$-Benzyl-4-methoxy- $\boldsymbol{N}$-tosylbenzamide (1c)}

Eluent: PE/EtOAc (85:15); white solid; yield: 269 mg (89\%); mp 121$123^{\circ} \mathrm{C}$.

${ }^{1} \mathrm{H}$ NMR $\left(\mathrm{CDCl}_{3}, 400 \mathrm{MHz}\right): \delta=2.40\left(\mathrm{~s}, 3 \mathrm{H}, \mathrm{CH}_{3}\right), 3.81\left(\mathrm{~s}, 3 \mathrm{H}, \mathrm{CH}_{3}\right)$, $4.88\left(\mathrm{~s}, 2 \mathrm{H}, \mathrm{CH}_{2}\right), 6.84(\mathrm{~d}, J=8.8 \mathrm{~Hz}, 2 \mathrm{H}, \mathrm{ArH}), 7.21-7.24(\mathrm{~m}, 7 \mathrm{H}$, $\mathrm{ArH}), 7.56(\mathrm{~d}, J=8.8 \mathrm{~Hz}, 2 \mathrm{H}, \mathrm{ArH}), 7.61(\mathrm{~d}, J=8.0 \mathrm{~Hz}, 2 \mathrm{H}, \mathrm{ArH})$.

${ }^{13} \mathrm{C} \mathrm{NMR}\left(\mathrm{CDCl}_{3}, 100 \mathrm{MHz}\right): \delta=21.58,51.33,55.40,113.47,127.12$, $127.68,128.02,128.32,128.49,129.41,131.26,135.82,136.12$, 144.48, 162.89, 171.26 .

HRMS: $m / z$ calcd for $\mathrm{C}_{22} \mathrm{H}_{21} \mathrm{NO}_{4} \mathrm{SNa}[\mathrm{M}+\mathrm{Na}]^{+}$: 418.1089; found: 418.1089.

\section{$\boldsymbol{N}$-Benzyl-4-bromo- $\boldsymbol{N}$-tosylbenzamide (1d)}

Eluent: PE/EtOAc (90:10); white solid; yield: $292 \mathrm{mg}$ (86\%); mp 131$133^{\circ} \mathrm{C}$.

${ }^{1} \mathrm{H}$ NMR $\left(\mathrm{CDCl}_{3}, 400 \mathrm{MHz}\right): \delta=2.41\left(\mathrm{~s}, 3 \mathrm{H}, \mathrm{CH}_{3}\right), 4.92\left(\mathrm{~s}, 2 \mathrm{H}, \mathrm{CH}_{2}\right)$, 7.20-7.26 (m, $7 \mathrm{H}, \mathrm{ArH}), 7.31$ (d, J = 8.4 Hz, $2 \mathrm{H}, \mathrm{ArH}$ ), 7.46 (d, $J=8.4$ $\mathrm{Hz}, 2 \mathrm{H}, \mathrm{ArH}), 7.56$ (d, J = 8.0 Hz, $2 \mathrm{H}, \mathrm{ArH})$.

${ }^{13} \mathrm{C} \mathrm{NMR}\left(\mathrm{CDCl}_{3}, 100 \mathrm{MHz}\right): \delta=21.62,50.98,126.57,127.86,127.95$, $128.33,128.60,129.51,129.89,131.37,133.90,135.60,135.92$, 144.91, 170.68 .

HRMS: $m / z$ calcd for $\mathrm{C}_{21} \mathrm{H}_{18} \mathrm{BrNO}_{3} \mathrm{SNa}[\mathrm{M}+\mathrm{Na}]^{+}: 466.0088$; found: 466.0094 .

\section{$N$-Benzyl-2-iodo- $N$-tosylbenzamide (1e)}

Eluent: PE/EtOAc (88:12); white solid; yield: 320 g (85\%); mp 146$148^{\circ} \mathrm{C}$.

${ }^{1} \mathrm{H} \mathrm{NMR}\left(\mathrm{CDCl}_{3}, 400 \mathrm{MHz}\right): \delta=2.42\left(\mathrm{~s}, 3 \mathrm{H}, \mathrm{CH}_{3}\right), 4.93\left(\mathrm{~s}, 2 \mathrm{H}, \mathrm{CH}_{2}\right)$, 7.23-7.33 (m, 9 H, ArH), 7.47 (d, $J=7.6 \mathrm{~Hz}, 2 \mathrm{H}, \operatorname{ArH}), 7.57$ (d, $J=7.2$ $\mathrm{Hz}, 2 \mathrm{H}, \mathrm{ArH})$.

${ }^{13} \mathrm{C}$ NMR $\left(\mathrm{CDCl}_{3}, 100 \mathrm{MHz}\right): \delta=21.63,51.01,126.56,127.27,127.86$, $127.96,128.34,128.39,128.61,129.53,129.89,129.99,131.38$, 133.91, 135.61, 135.95, 144.92, 170.67.

HRMS: $m / z$ calcd for $\mathrm{C}_{21} \mathrm{H}_{18} \mathrm{INO}_{3} \mathrm{SNa}[\mathrm{M}+\mathrm{Na}]^{+}:$513.9950; found: 513.9947.

\section{$\boldsymbol{N}$-Benzyl- $\mathbf{N}$-tosylfuran-2-carboxamide (1f) ${ }^{12}$}

Eluent: PE/EtOAc (85:15); light yellow solid; yield: $242 \mathrm{mg}$ (89\%); mp $113-115{ }^{\circ} \mathrm{C}$ 
${ }^{1} \mathrm{H}$ NMR $\left(\mathrm{CDCl}_{3}, 400 \mathrm{MHz}\right): \delta=2.45\left(\mathrm{~s}, 3 \mathrm{H}, \mathrm{CH}_{3}\right), 5.27\left(\mathrm{~s}, 2 \mathrm{H}, \mathrm{CH}_{2}\right)$, $6.48(\mathrm{~d}, J=2.0 \mathrm{~Hz}, 1 \mathrm{H}, \mathrm{ArH}), 7.15(\mathrm{~d}, J=3.2 \mathrm{~Hz}, 1 \mathrm{H}, \mathrm{ArH}), 7.25-7.30$ $(\mathrm{m}, 7 \mathrm{H}, \mathrm{ArH}), 7.52(\mathrm{~s}, 1 \mathrm{H}, \mathrm{ArH}), 7.80(\mathrm{~d}, J=8.0 \mathrm{~Hz}, 2 \mathrm{H}, \mathrm{ArH})$.

${ }^{13} \mathrm{C}$ NMR $\left(\mathrm{CDCl}_{3}, 100 \mathrm{MHz}\right): \delta=21.61,50.49,112.17,120.42,127.16$, $127.58,128.57,128.80,129.30,135.86,136.48,144.73,146.01$, $146.53,159.48$.

\section{N-Benzyl- $\mathbf{N}$-tosylthiophene-2-carboxamide (1g)}

Eluent: PE/EtOAc (85:15); white solid; yield: $259 \mathrm{mg} 91 \%$ ); $\mathrm{mp} 86-88^{\circ} \mathrm{C}$. ${ }^{1} \mathrm{H}$ NMR $\left(\mathrm{CDCl}_{3}, 400 \mathrm{MHz}\right): \delta=2.41\left(\mathrm{~s}, 3 \mathrm{H}, \mathrm{CH}_{3}\right), 5.06\left(\mathrm{~s}, 2 \mathrm{H}, \mathrm{CH}_{2}\right)$, 6.98 (t, $J=4.4 \mathrm{~Hz}, 1 \mathrm{H}, \mathrm{ArH}), 7.25-7.29(\mathrm{~m}, 7 \mathrm{H}, \mathrm{ArH}), 7.53$ (d, $J=4.4$ $\mathrm{Hz}, 2 \mathrm{H}, \mathrm{ArH}), 7.73$ (d, $J=8.0 \mathrm{~Hz}, 2 \mathrm{H}, \mathrm{ArH})$.

${ }^{13} \mathrm{C}$ NMR $\left(\mathrm{CDCl}_{3}, 100 \mathrm{MHz}\right): \delta=21.62,51.66,127.46,127.48,127.74$, $128.65,128.66,129.44,133.19,133.33,135.60,136.14,137.29$, 144.73, 164.66 .

HRMS: $m / z$ calcd for $\mathrm{C}_{19} \mathrm{H}_{17} \mathrm{NO}_{3} \mathrm{~S}_{2} \mathrm{Na}[\mathrm{M}+\mathrm{Na}]^{+}$: 394.0548; found: 394.0548 .

\section{$\mathbf{N}$-Benzyl-5-bromo- $\mathbf{N}$-tosylfuran-2-carboxamide (1h)}

Eluent: PE/EtOAc (80:20); white solid; yield: 283 mg (85\%); mp 119$121^{\circ} \mathrm{C}$.

${ }^{1} \mathrm{H} \mathrm{NMR}\left(\mathrm{CDCl}_{3}, 400 \mathrm{MHz}\right): \delta=2.41\left(\mathrm{~s}, 3 \mathrm{H}, \mathrm{CH}_{3}\right), 5.17\left(\mathrm{~s}, 2 \mathrm{H}, \mathrm{CH}_{2}\right)$, $6.36(\mathrm{~d}, J=3.6 \mathrm{~Hz}, 1 \mathrm{H}, \mathrm{ArH}), 7.02(\mathrm{~d}, J=3.6 \mathrm{~Hz}, 1 \mathrm{H}, \mathrm{ArH}), 7.20-7.29$ $(\mathrm{m}, 7 \mathrm{H}, \mathrm{ArH}), 7.78(\mathrm{~d}, J=8.0 \mathrm{~Hz}, 2 \mathrm{H}, \mathrm{ArH})$.

${ }^{13} \mathrm{C} \mathrm{NMR}\left(\mathrm{CDCl}_{3}, 100 \mathrm{MHz}\right): \delta=21.64,50.51,114.23,122.46,127.24$, $127.52,127.64,128.60,128.74,129.43,135.66,136.18,144.89$, $148.10,158.30$.

HRMS: $m / z$ calcd for $\mathrm{C}_{19} \mathrm{H}_{16} \mathrm{BrNO}_{4} \mathrm{SNa}[\mathrm{M}+\mathrm{Na}]^{+}$: 455.9881 ; found: 455.9881 .

\section{$\boldsymbol{N}$-Benzyl- $\boldsymbol{N}$-tosylacetamide (1i) $)^{13}$}

Eluent: PE/EtOAc (90:10); colorless oil; yield: $195 \mathrm{mg}$ (84\%).

${ }^{1} \mathrm{H} \mathrm{NMR}\left(\mathrm{CDCl}_{3}, 400 \mathrm{MHz}\right): \delta=2.32\left(\mathrm{~s}, 3 \mathrm{H}, \mathrm{CH}_{3}\right), 2.45\left(\mathrm{~s}, 3 \mathrm{H}, \mathrm{CH}_{3}\right)$, $5.11\left(\mathrm{~s}, 2 \mathrm{H}, \mathrm{CH}_{2}\right) 7.29-7.36(\mathrm{~m}, 6 \mathrm{H}, \mathrm{ArH}), 7.39(\mathrm{t}, J=7.6 \mathrm{~Hz}, 2 \mathrm{H}, \mathrm{ArH})$, $7.64(\mathrm{~d}, J=8.4 \mathrm{~Hz}, 2 \mathrm{H}, \mathrm{ArH})$.

${ }^{13} \mathrm{C} \mathrm{NMR}\left(\mathrm{CDCl}_{3}, 100 \mathrm{MHz}\right): \delta=21.56,24.86,49.48,127.71,127.83$, $127.93,128.57,128.66,129.71,136.49,136.63,144.88,170.33$.

\section{4-Methoxy- $\mathrm{N}$-methyl- $\mathrm{N}$-tosylbenzamide (4a) ${ }^{14}$}

Eluent: PE/EtOAC (90:10); white solid; yield: $785 \mathrm{mg}$ (91\%); mp $46-48{ }^{\circ} \mathrm{C}$. ${ }^{1} \mathrm{H} \mathrm{NMR}\left(\mathrm{CDCl}_{3}, 400 \mathrm{MHz}\right): \delta=2.42\left(\mathrm{~s}, 3 \mathrm{H}, \mathrm{CH}_{3}\right), 3.24\left(\mathrm{~s}, 3 \mathrm{H}, \mathrm{NCH}_{3}\right)$, $3.83\left(\mathrm{~s}, 3 \mathrm{H}, \mathrm{OCH}_{3}\right), 7.89$ (d, J = 7.6 Hz, $\left.2 \mathrm{H}, \mathrm{ArH}\right), 7.32$ (d, J = 7.6 Hz, $2 \mathrm{H}$, $\operatorname{ArH}), 7.61(\mathrm{~d}, J=7.6 \mathrm{~Hz}, 2 \mathrm{H}, \mathrm{ArH}), 7.84(\mathrm{~d}, J=8.0 \mathrm{~Hz}, 2 \mathrm{H}, \mathrm{ArH})$.

${ }^{13} \mathrm{C}$ NMR $\left(\mathrm{CDCl}_{3}, 100 \mathrm{MHz}\right): \delta=21.61,35.90,55.44,113.55,126.24$, $128.35,129.57,131.30,134.97,144.74,162.91,171.20$.

\section{$\mathbf{N}$-Methyl-4-nitro- $\boldsymbol{N}$-tosylbenzamide (4b) $)^{14}$}

Eluent: PE/EtOAc (85:15); light yellow solid; yield: 794 mg (88\%); mp $114-116{ }^{\circ} \mathrm{C}$.

${ }^{1} \mathrm{H} \mathrm{NMR}\left(\mathrm{CDCl}_{3}, 400 \mathrm{MHz}\right): \delta=2.47\left(\mathrm{~s}, 3 \mathrm{H}, \mathrm{CH}_{3}\right), 3.27\left(\mathrm{~s}, 3 \mathrm{H}, \mathrm{NCH}_{3}\right)$, $7.36(\mathrm{~d}, J=8.4 \mathrm{~Hz}, 2 \mathrm{H}, \mathrm{ArH}), 7.69(\mathrm{t}, J=9.6 \mathrm{~Hz}, 4 \mathrm{H}, \mathrm{ArH}), 8.26$ (d, $J=8.8 \mathrm{~Hz}, 2 \mathrm{H}, \mathrm{ArH})$.

${ }^{13} \mathrm{C} \mathrm{NMR}\left(\mathrm{CDCl}_{3}, 100 \mathrm{MHz}\right): \delta=21.67,34.62,123.32,128.01,129.16$, $129.95,134.55,140.88,145.59,149.28,169.55$.

\section{$\mathbf{N}$-Methyl- $\mathbf{N}$-tosylbenzamide (4c) ${ }^{14}$}

Eluent: PE/EtOAC (85:15); white solid; yield: $672 \mathrm{mg}$ (86\%); mp 65-67 ${ }^{\circ} \mathrm{C}$. ${ }^{1} \mathrm{H} \mathrm{NMR}\left(\mathrm{CDCl}_{3}, 400 \mathrm{MHz}\right): \delta=2.42\left(\mathrm{~s}, 3 \mathrm{H}, \mathrm{CH}_{3}\right), 3.27\left(\mathrm{~s}, 3 \mathrm{H}, \mathrm{NCH}_{3}\right)$, $7.32(\mathrm{~d}, 2 \mathrm{H}, J=8.0 \mathrm{~Hz}, \mathrm{ArH}), 7.39(\mathrm{t}, 2 \mathrm{H}, J=7.6 \mathrm{~Hz}, \mathrm{ArH}), 7.49$ (dd, $\left.J_{1}=7.0 \mathrm{~Hz}, J_{2}=1.2 \mathrm{~Hz}, 1 \mathrm{H}, \mathrm{ArH}\right), 7.53\left(\mathrm{dd}, J_{1}=7.8 \mathrm{~Hz}, J_{2}=1.2 \mathrm{~Hz}, 2 \mathrm{H}\right.$, ArH), $7.83(\mathrm{~d}, J=7.0 \mathrm{~Hz}, 2 \mathrm{H}, \mathrm{ArH})$.

${ }^{13} \mathrm{C} \mathrm{NMR}\left(\mathrm{CDCl}_{3}, 100 \mathrm{MHz}\right): \delta=21.63,35.61,128.28,128.38,128.43$, 129.61, 130.09, 131.94, 134.47, 135.18, 144.93, 171.45 .

\section{$\boldsymbol{N}$-Phenyl- $\boldsymbol{N}$-tosylbenzamide (4d) ${ }^{8 \mathrm{~d}}$}

Eluent: PE/EtOAc (85:15); white solid; yield: 2.44 g (86\%); mp 151$153^{\circ} \mathrm{C}$.

${ }^{1} \mathrm{H}$ NMR $\left(\mathrm{CDCl}_{3}, 400 \mathrm{MHz}\right): \delta=2.43\left(\mathrm{~s}, 3 \mathrm{H}, \mathrm{CH}_{3}\right), 7.14-7.15(\mathrm{~m}, 3 \mathrm{H}$, ArH), 7.26-7.30 (m, $5 \mathrm{H}, \mathrm{ArH}), 7.42(\mathrm{~d}, J=7.2 \mathrm{~Hz}, 2 \mathrm{H}, \mathrm{ArH}), 7.82(\mathrm{~d}$, $J=8.4 \mathrm{~Hz}, 2 \mathrm{H}, \mathrm{ArH})$.

${ }^{13} \mathrm{C} \mathrm{NMR}\left(\mathrm{CDCl}_{3}, 100 \mathrm{MHz}\right): \delta=21.67,127.95,129.01,129.07,129.22$, $129.42,130.35,131.71,133.60,135.17,137.36,144.81,169.87$.

\section{N-Boc-Activated Secondary Amides; General Procedure}

To an oven-dried $100 \mathrm{~mL}$ round-bottomed flask containing a secondary amide substrate ( $1.0 \mathrm{mmol}, 1.0$ equiv) and DMAP $(0.1 \mathrm{mmol}, 0.1$ equiv) in $\mathrm{CH}_{2} \mathrm{Cl}_{2}\left(20 \mathrm{~mL}\right.$ ) was added $\mathrm{Boc}_{2} \mathrm{O}$ (1.3 mmol, 1.3 equiv) in one portion and the reaction mixture was allowed to stir at r.t. After the indicated time, the mixture was quenched with aq $\mathrm{NaHCO}_{3}(10$ $\mathrm{mL})$, and extracted with EtOAc $(3 \times 20 \mathrm{~mL})$. the combined organic layers were washed with $\mathrm{H}_{2} \mathrm{O}(20 \mathrm{~mL})$ and brine $(20 \mathrm{~mL})$, dried (anhyd $\mathrm{Na}_{2} \mathrm{SO}_{4}$ ), and concentrated. The crude product was purified by column chromatography (EtOAc/PE) to afford the pure product.

\section{tert-Butyl (4-Methoxybenzoyl)(methyl)carbamate (6a) $)^{8 \mathrm{~d}}$}

Eluent: PE/EtOAc (85:15); colorless oil; yield: $715 \mathrm{mg}$ (89\%).

${ }^{1} \mathrm{H} \mathrm{NMR}\left(\mathrm{CDCl}_{3}, 400 \mathrm{MHz}\right): \delta=1.19\left(\mathrm{~s}, 9 \mathrm{H}, 3 \times \mathrm{CH}_{3}\right), 3.24(\mathrm{~s}, 3 \mathrm{H}$, $\left.\mathrm{NCH}_{3}\right), 3.81\left(\mathrm{~s}, 3 \mathrm{H}, \mathrm{OCH}_{3}\right), 6.85(\mathrm{~d}, J=8.8 \mathrm{~Hz}, 2 \mathrm{H}, \mathrm{ArH}), 7.49(\mathrm{~d}, J=8.8$ $\mathrm{Hz}, 2 \mathrm{H}, \mathrm{ArH})$.

${ }^{13} \mathrm{C}$ NMR $\left(\mathrm{CDCl}_{3}, 100 \mathrm{MHz}\right): \delta=27.46,32.75,55.36,82.58,133.14$, $129.69,129.90,153.77,162.06,173.13$.

tert-Butyl Methyl[4-(trifluoromethyl)benzoyl]carbamate (6b) $)^{8 \mathrm{~d}}$ Eluent: PE/EtOAc (90:10); white solid; yield: 627 mg (84\%); mp 147$149^{\circ} \mathrm{C}$.

${ }^{1} \mathrm{H}$ NMR $\left(\mathrm{CDCl}_{3}, 400 \mathrm{MHz}\right): \delta=1.16\left(\mathrm{~s}, 9 \mathrm{H}, 3 \times \mathrm{CH}_{3}\right), 3.31(\mathrm{~s}, 3 \mathrm{H}$, $\left.\mathrm{NCH}_{3}\right), 7.57(\mathrm{~d}, J=8.0 \mathrm{~Hz}, 2 \mathrm{H}, \mathrm{ArH}), 7.64(\mathrm{~d}, J=8.0 \mathrm{~Hz}, 2 \mathrm{H}, \mathrm{ArH})$.

${ }^{13} \mathrm{C} \mathrm{NMR}\left(\mathrm{CDCl}_{3}, 100 \mathrm{MHz}\right): \delta=27.33,32.37,83.63,124.98,127.40$, $141.29,152.92,172.04$.

\section{tert-Butyl Benzoyl(benzyl)carbamate (11a) ${ }^{8 \mathrm{~d}}$}

Eluent: PE/EtOAc (90:10); colorless oil; yield: $1.31 \mathrm{~g}$ (89\%).

${ }^{1} \mathrm{H} \mathrm{NMR}\left(\mathrm{CDCl}_{3}, 400 \mathrm{MHz}\right): \delta=1.11\left(\mathrm{~s}, 9 \mathrm{H}, 3 \times \mathrm{CH}_{3}\right), 4.99\left(\mathrm{~s}, 2 \mathrm{H}, \mathrm{CH}_{2}\right)$, $7.24(\mathrm{t}, J=6.4 \mathrm{~Hz}, 1 \mathrm{H}, \mathrm{ArH}), 7.29-7.37(\mathrm{~m}, 4 \mathrm{H}, \mathrm{ArH}), 7.42(\mathrm{t}, J=6.0 \mathrm{~Hz}$, $3 \mathrm{H}, \mathrm{ArH}), 7.50$ (d, $J=7.2 \mathrm{~Hz}, 2 \mathrm{H}, \mathrm{ArH})$.

${ }^{13} \mathrm{C} \mathrm{NMR}\left(\mathrm{CDCl}_{3}, 100 \mathrm{MHz}\right): \delta=27.31,48.84,83.10,127.38,127.44$, 128.04, 128.14, 128.43, 131.02, 137.71, 137.86, 153.42, 173.03 .

tert-Butyl Benzyl(4-bromobenzoyl)carbamate (11b)

Eluent: PE/EtOAc (90:10); colorless oil; yield: $504 \mathrm{mg}$ (75\%). 
${ }^{1} \mathrm{H} \mathrm{NMR}\left(\mathrm{CDCl}_{3}, 400 \mathrm{MHz}\right): \delta=1.16\left(\mathrm{~s}, 9 \mathrm{H}, 3 \times \mathrm{CH}_{3}\right), 4.95\left(\mathrm{~s}, 2 \mathrm{H}, \mathrm{CH}_{2}\right)$, 7.25-7.27 (m, $2 \mathrm{H}, \mathrm{ArH}), 7.32(\mathrm{t}, J=7.6 \mathrm{~Hz}, 2 \mathrm{H}, \mathrm{ArH}), 7.38(\mathrm{t}, J=6.4 \mathrm{~Hz}$, $3 \mathrm{H}, \mathrm{ArH}), 7.52\left(\mathrm{dd}, J_{1}=7.8 \mathrm{~Hz}, J_{2}=1.2 \mathrm{~Hz}, 2 \mathrm{H}, \mathrm{ArH}\right)$.

${ }^{13} \mathrm{C}$ NMR $\left(\mathrm{CDCl}_{3}, 100 \mathrm{MHz}\right): \delta=27.39,48.88,83.53,125.51,127.46$, 128.06, 128.45, 129.02, 131.26, 136.40, 137.60, 153.16, 172.07 .

HRMS: $m / z$ calcd for $\mathrm{C}_{19} \mathrm{H}_{20} \mathrm{BrNO}_{3} \mathrm{Na}[\mathrm{M}+\mathrm{Na}]^{+}:$412.0524; found: 412.0527 .

\section{Transamidation of Activated Amides; General Procedure}

The amide substrate $\mathbf{1}, \mathbf{4}, \mathbf{6}, \mathbf{1 0}$, or $\mathbf{1 1}$ (1.0 mmol, 1.0 equiv) and the respective amine $\mathbf{2}(1.3 \mathrm{mmol}, 1.3$ equiv) in $\mathrm{EtOH}(1 \mathrm{~mL})$ were taken in a vial and the reaction mixture was stirred at $45^{\circ} \mathrm{C}$. The reaction was monitored by TLC. After completion of the reaction, the mixture was concentrated in vacuo and the residue was purified by column chromatography using silica gel (EtOAc/PE) to give the corresponding transamidation product.

\section{Phenyl(piperidin-1-yl)methanone (3a) ${ }^{8 \mathrm{~d}}$}

Eluent: PE/EtOAc (75:25); colorless oil; yield: $51.3 \mathrm{mg}$ (99\%).

${ }^{1} \mathrm{H} \mathrm{NMR}\left(\mathrm{CDCl}_{3}, 400 \mathrm{MHz}\right): \delta=1.47\left(\mathrm{~s}, 2 \mathrm{H}, \mathrm{CH}_{2}\right), 1.63\left(\mathrm{~s}, 4 \mathrm{H}, \mathrm{CH}_{2}\right)$, $3.29\left(\mathrm{~s}, 2 \mathrm{H}, \mathrm{CH}_{2}\right), 3.67$ (s, $\left.2 \mathrm{H}, \mathrm{CH}_{2}\right), 7.35$ (d, J = 4.0 Hz, $5 \mathrm{H}, \mathrm{ArH}$ ).

${ }^{13} \mathrm{C}$ NMR $\left(\mathrm{CDCl}_{3}, 100 \mathrm{MHz}\right): \delta=24.51,25.57,26.46,29.62,43.03$, $48.69,126.70,128.32,129.27,136.43,170.22$.

\section{Morpholino(phenyl)methanone (3b) ${ }^{8 \mathrm{~d}}$}

Eluent: PE/EtOAc (85:15); colorless oil; yield: $50.7 \mathrm{mg}$ (97\%).

${ }^{1} \mathrm{H} \mathrm{NMR}\left(\mathrm{CDCl}_{3}, 400 \mathrm{MHz}\right): \delta=2.28-3.59\left(\mathrm{~m}, 8 \mathrm{H}, \mathrm{CH}_{2}\right), 7.26(\mathrm{~d}, J=7.6$ $\mathrm{Hz}, 5 \mathrm{H}, \mathrm{ArH})$.

${ }^{13} \mathrm{C} \mathrm{NMR}\left(\mathrm{CDCl}_{3}, 100 \mathrm{MHz}\right): \delta=42.45,48.04,66.66,126.98,128.12$, $128.41,129.75,132.64,135.12,170.26$.

\section{Phenyl(pyrrolidin-1-yl)methanone (3c) ${ }^{8 \mathrm{~d}}$}

Eluent: PE/EtOAc (75:25); colorless oil; yield: $47.5 \mathrm{mg}$ (99\%).

${ }^{1} \mathrm{H}$ NMR $\left(\mathrm{CDCl}_{3}, 400 \mathrm{MHz}\right): \delta=1.68-1.73\left(\mathrm{~m}, 2 \mathrm{H}, \mathrm{CH}_{2}\right), 1.76-1.81(\mathrm{~m}$, $\left.2 \mathrm{H}, \mathrm{CH}_{2}\right), 3.26\left(\mathrm{t}, 2 \mathrm{H}, J=6.4 \mathrm{~Hz}, \mathrm{CH}_{2}\right), 3.50\left(\mathrm{t}, J=6.8 \mathrm{~Hz}, 2 \mathrm{H}, \mathrm{CH}_{2}\right.$ ), 7.23-7.25 (m, $3 \mathrm{H}, \mathrm{ArH}), 7.37-7.39$ (m, $2 \mathrm{H}, \mathrm{ArH})$.

${ }^{13} \mathrm{C} \mathrm{NMR}\left(\mathrm{CDCl}_{3}, 100 \mathrm{MHz}\right): \delta=24.29,26.22,46.08,49.49,126.95$, $128.03,128.09,129.67,130.81,132.43,136.95,169.60$.

\section{Piperidin-1-yl(p-tolyl)methanone (3d) ${ }^{15}$}

Eluent: PE/EtOAc (85:15); colorless oil; yield: $53.4 \mathrm{mg}$ (96\%).

${ }^{1} \mathrm{H}$ NMR $\left(\mathrm{CDCl}_{3}, 400 \mathrm{MHz}\right): \delta=1.44\left(\mathrm{~s}, 2 \mathrm{H}, \mathrm{CH}_{2}\right), 1.59\left(\mathrm{~s}, 4 \mathrm{H}, \mathrm{CH}_{2}\right)$, $2.29\left(\mathrm{~s}, 3 \mathrm{H}, \mathrm{CH}_{3}\right), 3.29\left(\mathrm{~s}, 2 \mathrm{H}, \mathrm{CH}_{2}\right), 3.63\left(\mathrm{~s}, 2 \mathrm{H}, \mathrm{CH}_{2}\right), 7.12(\mathrm{~d}, J=6.8$ $\mathrm{Hz}, 2 \mathrm{H}, \mathrm{ArH}), 7.22$ (dd, $\left.J_{1}=7.4 \mathrm{~Hz}, J_{2}=1.2 \mathrm{~Hz}, 2 \mathrm{H}, \mathrm{ArH}\right)$.

${ }^{13} \mathrm{C} \mathrm{NMR}\left(\mathrm{CDCl}_{3}, 100 \mathrm{MHz}\right): \delta=21.29,24.53,25.59,26.43,43.11$, $48.75,126.83,128.90,133.40,139.33,170.44$.

\section{(4-Methoxyphenyl)(morpholino)methanone (3e) ${ }^{16}$}

Eluent: PE/EtOAc (85:15); colorless oil; yield: $53.1 \mathrm{mg}$ (95\%).

${ }^{1} \mathrm{H} \mathrm{NMR}\left(\mathrm{CDCl}_{3}, 400 \mathrm{MHz}\right): \delta=3.66\left(\mathrm{~m}, 8 \mathrm{H}, \mathrm{CH}_{2}\right), 3.79\left(\mathrm{~s}, 3 \mathrm{H}, \mathrm{OCH}_{3}\right)$, 6.87-6.89 (m, 2 H, ArH), 7.34-7.36 (m, 2 H, ArH).

${ }^{13} \mathrm{C}$ NMR $\left(\mathrm{CDCl}_{3}, 100 \mathrm{MHz}\right): \delta=55.31,66.87,113.72,127.25,129.15$, $160.83,170.35$.

\section{(4-Bromophenyl)(piperidin-1-yl)methanone (3f) ${ }^{17}$}

Eluent: PE/EtOAc (85:15); white solid; yield: $56.5 \mathrm{mg}$ (94\%); mp 93$95{ }^{\circ} \mathrm{C}$.
${ }^{1} \mathrm{H}$ NMR $\left(\mathrm{CDCl}_{3}, 400 \mathrm{MHz}\right): \delta=1.47\left(\mathrm{~s}, 2 \mathrm{H}, \mathrm{CH}_{2}\right), 1.63\left(\mathrm{~s}, 4 \mathrm{H}, \mathrm{CH}_{2}\right)$, $3.28\left(\mathrm{~s}, 2 \mathrm{H}, \mathrm{CH}_{2}\right), 3.65\left(\mathrm{~s}, 2 \mathrm{H}, \mathrm{CH}_{2}\right), 7.23\left(\mathrm{dd}, J_{1}=8.0 \mathrm{~Hz}, J_{2}=0.8 \mathrm{~Hz}, 2\right.$ $\mathrm{H}, \mathrm{ArH}), 7.49\left(\mathrm{dd}, J_{1}=8.0 \mathrm{~Hz}, J_{2}=0.8 \mathrm{~Hz}, 2 \mathrm{H}, \mathrm{ArH}\right)$.

${ }^{13} \mathrm{C}$ NMR $\left(\mathrm{CDCl}_{3}, 100 \mathrm{MHz}\right): \delta=24.46,25.55,43.19,48.72,123.54$, $128.52,131.57,135.25,169.16$

\section{(4-Bromophenyl)(morpholino)methanone (3g) ${ }^{16}$}

Eluent: PE/EtOAc (85:15); colorless oil; yield: $55.3 \mathrm{mg}$ (91\%).

${ }^{1} \mathrm{H} \mathrm{NMR}\left(\mathrm{CDCl}_{3}, 400 \mathrm{MHz}\right): \delta=3.44-3.71\left(\mathrm{~m}, 8 \mathrm{H}, \mathrm{CH}_{2}\right), 7.29(\mathrm{~d}, J=8.0$ $\mathrm{Hz}, 2 \mathrm{H}, \mathrm{ArH}), 7.55$ (d, J = 8.0 Hz, $2 \mathrm{H}, \mathrm{ArH})$.

${ }^{13} \mathrm{C}$ NMR $\left(\mathrm{CDCl}_{3}, 100 \mathrm{MHz}\right): \delta=42.60,48.09,66.74,124.14,128.85$, $131.75,134.09,169.25$

\section{(2-Iodophenyl)(piperidin-1-yl)methanone (3h) ${ }^{18}$}

Eluent: PE/EtOAc (85:15); colorless oil; yield: $59.7 \mathrm{mg}$ (93\%).

${ }^{1} \mathrm{H} \mathrm{NMR}\left(\mathrm{CDCl}_{3}, 400 \mathrm{MHz}\right): \delta=1.41-1.71\left(\mathrm{~m}, 6 \mathrm{H}, \mathrm{CH}_{2}\right), 3.08-3.21(\mathrm{~m}$, $\left.2 \mathrm{H}, \mathrm{CH}_{2}\right), 3.65-3.79\left(\mathrm{~m}, 2 \mathrm{H}, \mathrm{CH}_{2}\right), 7.03\left(\mathrm{td}, J_{1}=7.4 \mathrm{~Hz}, J_{2}=2.0 \mathrm{~Hz}, 1 \mathrm{H}\right.$, ArH), $7.16\left(\mathrm{dd}, J_{1}=7.6 \mathrm{~Hz}, J_{2}=1.6 \mathrm{~Hz}, 1 \mathrm{H}, \mathrm{ArH}\right), 7.35\left(\mathrm{td}, J_{1}=7.6 \mathrm{~Hz}\right.$, $\left.J_{2}=0.8 \mathrm{~Hz}, 1 \mathrm{H}, \mathrm{ArH}\right), 7.79$ (dd, $\left.J_{1}=7.6 \mathrm{~Hz}, J_{2}=0.8 \mathrm{~Hz}, 1 \mathrm{H}, \mathrm{ArH}\right)$.

${ }^{13} \mathrm{C}$ NMR $\left(\mathrm{CDCl}_{3}, 100 \mathrm{MHz}\right): \delta=24.45,25.36,26.22,42.46,47.91$, $92.47,126.78,128.27,129.90,139.13,142.76,169.14$.

\section{(2-Iodophenyl)(morpholino)methanone (3i) ${ }^{19}$}

Eluent: PE/EtOAc (85:15); white solid; yield: 59.4 mg (91\%); mp 82$84^{\circ} \mathrm{C}$.

${ }^{1} \mathrm{H} \mathrm{NMR}\left(\mathrm{CDCl}_{3}, 400 \mathrm{MHz}\right): \delta=3.15\left(\mathrm{~s}, 1 \mathrm{H}, \mathrm{CH}_{2}\right), 3.22\left(\mathrm{~s}, 1 \mathrm{H}, \mathrm{CH}_{2}\right)$, $3.55\left(\mathrm{~s}, 1 \mathrm{H}, \mathrm{CH}_{2}\right), 3.72-3.79\left(\mathrm{~m}, 5 \mathrm{H}, \mathrm{CH}_{2}\right), 7.04(\mathrm{t}, J=7.6 \mathrm{~Hz}, 1 \mathrm{H}, \mathrm{ArH})$, $7.16(\mathrm{~d}, J=7.2 \mathrm{~Hz}, 1 \mathrm{H}, \mathrm{ArH}), 7.35(\mathrm{t}, J=7.2 \mathrm{~Hz}, 1 \mathrm{H}, \mathrm{ArH}), 7.89$ (t, $J=7.6$ $\mathrm{Hz}, 1 \mathrm{H}, \mathrm{ArH})$.

${ }^{13} \mathrm{C}$ NMR $\left(\mathrm{CDCl}_{3}, 100 \mathrm{MHz}\right): \delta=41.95,47.20,66.52,66.63,92.38$, $127.04,128.45,130.38,139.20,141.62,169.38$.

\section{Furan-2-yl(piperidin-1-yl)methanone $(3 \mathbf{j})^{20}$}

Eluent: PE/EtOAc (85:15); white solid; yield: $48.9 \mathrm{mg}$ (97\%); mp 46$48^{\circ} \mathrm{C}$.

${ }^{1} \mathrm{H}$ NMR $\left(\mathrm{CDCl}_{3}, 400 \mathrm{MHz}\right): \delta=1.48-1.55\left(\mathrm{~m}, 6 \mathrm{H}, \mathrm{CH}_{2}\right), 3.55(\mathrm{~s}, 4 \mathrm{H}$, $\left.\mathrm{CH}_{2}\right), 6.31-6.33(\mathrm{~m}, 1 \mathrm{H}, \mathrm{ArH}), 6.79\left(\mathrm{dd}, J_{1}=3.4 \mathrm{~Hz}, J_{2}=0.8 \mathrm{~Hz}, 1 \mathrm{H}\right.$, ArH), 7.34 (d, $J=0.8 \mathrm{~Hz}, 1 \mathrm{H}, \mathrm{ArH})$.

${ }^{13} \mathrm{C}$ NMR $\left(\mathrm{CDCl}_{3}, 100 \mathrm{MHz}\right): \delta=24.51,25.99,29.52,43.88,47.57$, $110.94,115.38,143.34,147.94,159.10$.

\section{(5-Bromofuran-2-yl)(piperidin-1-yl)methanone (3k) $)^{21}$}

Eluent: PE/EtOAc (85:15); white solid; yield: $57.1 \mathrm{mg}$ (96\%); mp 53$55{ }^{\circ} \mathrm{C}$.

${ }^{1} \mathrm{H} \mathrm{NMR}\left(\mathrm{CDCl}_{3}, 400 \mathrm{MHz}\right): \delta=1.61-1.69\left(\mathrm{~m}, 6 \mathrm{H}, \mathrm{CH}_{2}\right), 3.65(\mathrm{~s}, 4 \mathrm{H}$, $\left.\mathrm{CH}_{2}\right), 6.37(\mathrm{~d}, J=3.6 \mathrm{~Hz}, 1 \mathrm{H}, \mathrm{ArH}), 6.88(\mathrm{~d}, J=3.6 \mathrm{~Hz}, 1 \mathrm{H}, \mathrm{ArH})$.

${ }^{13} \mathrm{C}$ NMR $\left(\mathrm{CDCl}_{3}, 100 \mathrm{MHz}\right): \delta=24.57,25.71,29.65,113.05,118.03$, $123.71,149.85,157.91$

\section{Piperidin-1-yl(thiophen-2-yl)methanone (31) ${ }^{20}$}

Eluent: PE/EtOAc (85:15); white solid; yield: $50.9 \mathrm{mg}$ (96\%); mp 55-57 ${ }^{\circ} \mathrm{C}$. ${ }^{1} \mathrm{H}$ NMR $\left(\mathrm{CDCl}_{3}, 400 \mathrm{MHz}\right): \delta=1.59-1.60\left(\mathrm{~m}, 4 \mathrm{H}, \mathrm{CH}_{2}\right), 1.65-1.67(\mathrm{~m}$, $\left.2 \mathrm{H}, \mathrm{CH}_{2}\right), 3.61-3.64\left(\mathrm{~m}, 4 \mathrm{H}, \mathrm{CH}_{2}\right), 6.99\left(\mathrm{td}, J_{1}=4.0 \mathrm{~Hz}, J_{2}=0.8 \mathrm{~Hz}, 1 \mathrm{H}\right.$, $\operatorname{ArH}), 7.22(\mathrm{~d}, J=3.2 \mathrm{~Hz}, 1 \mathrm{H}, \mathrm{ArH}), 7.38(\mathrm{~d}, J=5.2 \mathrm{~Hz}, 1 \mathrm{H}, \mathrm{ArH})$.

${ }^{13} \mathrm{C} \mathrm{NMR}\left(\mathrm{CDCl}_{3}, 100 \mathrm{MHz}\right): \delta=24.56,26.08,26.29,29.63,126.49$, $128.03,128.20,137.56,163.41$. 


\section{$\mathbf{N}$-Methyl- $\mathbf{N}$-phenylbenzamide ( $3 \mathrm{~m})^{15}$}

Eluent: PE/EtOAc (90:10); white solid; yield: $54.9 \mathrm{mg}$ (95\%); mp 61$63{ }^{\circ} \mathrm{C}$.

${ }^{1} \mathrm{H} \mathrm{NMR}\left(\mathrm{CDCl}_{3}, 400 \mathrm{MHz}\right): \delta=3.46\left(\mathrm{~s}, 3 \mathrm{H}, \mathrm{CH}_{3}\right), 6.99(\mathrm{~d}, J=7.6 \mathrm{~Hz}, 2$ $\mathrm{H}, \operatorname{ArH}), 7.11(\mathrm{t}, J=8.0 \mathrm{~Hz}, 3 \mathrm{H}, \mathrm{ArH}), 7.16-7.17(\mathrm{~m}, 3 \mathrm{H}, \operatorname{ArH}), 7.26(\mathrm{~d}$, $J=7.6 \mathrm{~Hz}, 2 \mathrm{H}$, ArH).

${ }^{13} \mathrm{C}$ NMR $\left(\mathrm{CDCl}_{3}, 100 \mathrm{MHz}\right): \delta=38.37,126.47,126.85,127.68,128.65$, $129.10,129.56,135.82,144.79,170.68$.

\section{$\boldsymbol{N}$-Phenylbenzamide $(3 n)^{7 a}$}

Eluent: PE/EtOAc (70:30); brownish solid; yield: $51.8 \mathrm{mg}$ (96\%); mp $160-162{ }^{\circ} \mathrm{C}$.

${ }^{1} \mathrm{H} \mathrm{NMR}\left(\mathrm{CDCl}_{3}, 400 \mathrm{MHz}\right): \delta=7.14\left(\mathrm{td}, J_{1}=7.0 \mathrm{~Hz}, J_{2}=0.4 \mathrm{~Hz}, 1 \mathrm{H}\right.$, $\mathrm{ArH}), 7.35(\mathrm{t}, J=7.6 \mathrm{~Hz}, 2 \mathrm{H}, \mathrm{ArH}), 7.45(\mathrm{t}, J=8.0 \mathrm{~Hz}, 2 \mathrm{H}, \mathrm{ArH}), 7.52$ $\left(\mathrm{td}, J_{1}=7.4 \mathrm{~Hz}, J_{2}=0.4 \mathrm{~Hz}, 1 \mathrm{H}, \mathrm{ArH}\right), 7.63(\mathrm{~d}, J=8.0 \mathrm{~Hz}, 2 \mathrm{H}, \mathrm{ArH}), 7.85$ (t, $J=7.6 \mathrm{~Hz}, 2 \mathrm{H}, \mathrm{ArH}), 7.98(\mathrm{~s}, 1 \mathrm{H}, \mathrm{NH})$.

${ }^{13} \mathrm{C}$ NMR $\left(\mathrm{CDCl}_{3}, 100 \mathrm{MHz}\right): \delta=120.26,124.56,127.02,128.41$, $128.73,129.04,130.12,131.80,134.91,137.87,165.87$.

\section{$\mathbf{N}$-(4-Iodophenyl)benzamide (3o) $)^{22}$}

Eluent: PE/EtOAc (70:30); white solid; yield: $81.3 \mathrm{mg}$ (92\%); mp 214$216{ }^{\circ} \mathrm{C}$.

${ }^{1} \mathrm{H}$ NMR (DMSO- $\left.d_{6}, 400 \mathrm{MHz}\right): \delta=7.46-7.41(\mathrm{~m}, 2 \mathrm{H}, \mathrm{ArH}$ ), 7.54-7.59 $(\mathrm{m}, 3 \mathrm{H}, \mathrm{ArH}), 7.65$ (d, $J=8.8 \mathrm{~Hz}, 2 \mathrm{H}, \mathrm{ArH}), 7.89(\mathrm{~d}, J=7.2 \mathrm{~Hz}, 2 \mathrm{H}$, ArH), 10.32 (s, $1 \mathrm{H}, \mathrm{NH})$.

${ }^{13} \mathrm{C}$ NMR (DMSO- $d_{6}, 100 \mathrm{MHz}$ ): $\delta=122.96,128.05,128.86,128.99$, $129.66,132.17,135.03,137.69,139.56,166.15$

\section{$\mathbf{N}$-(3,5-Dichlorophenyl)benzamide (3p) $)^{23}$}

Eluent: PE/EtOAc (70:30); white solid; yield: 65.5 mg (90\%); mp 148$150{ }^{\circ} \mathrm{C}$.

${ }^{1} \mathrm{H} \mathrm{NMR}\left(\mathrm{CDCl}_{3}, 400 \mathrm{MHz}\right): \delta=7.46\left(\mathrm{td}, J_{1}=7.4 \mathrm{~Hz}, J_{2}=0.4 \mathrm{~Hz}, 3 \mathrm{H}\right.$, $\mathrm{ArH}), 7.61\left(\mathrm{td}, J_{1}=6.6 \mathrm{~Hz}, J_{2}=1.2 \mathrm{~Hz}, 2 \mathrm{H}, \mathrm{ArH}\right), 8.13\left(\mathrm{dd}, 3 \mathrm{H}, J_{1}=7.8\right.$ $\left.\mathrm{Hz}, J_{2}=1.2 \mathrm{~Hz}, \mathrm{ArH}\right), 10.60$ (br, $1 \mathrm{H}, \mathrm{NH}$ ).

${ }^{13} \mathrm{C}$ NMR $\left(\mathrm{CDCl}_{3}, 100 \mathrm{MHz}\right): \delta=128.46,129.29,130.18,133.79$, 172.42 .

\section{$N$-Benzylbenzamide (3q) $)^{8 \mathrm{~d}}$}

Eluent: PE/EtOAc (75:25); white solid; yield: 56.6 mg (98\%); mp 105$107^{\circ} \mathrm{C}$.

${ }^{1} \mathrm{H} \mathrm{NMR}\left(\mathrm{CDCl}_{3}, 400 \mathrm{MHz}\right): \delta=4.64(\mathrm{~d}, J=5.6 \mathrm{~Hz}, 2 \mathrm{H}, \mathrm{ArH}), 6.57(\mathrm{~s}, 1$ $\mathrm{H}, \mathrm{NH}), 7.23-7.36(\mathrm{~m}, 5 \mathrm{H}, \mathrm{ArH}), 7.42\left(\mathrm{td}, J_{1}=7.2 \mathrm{~Hz}, J_{2}=1.2 \mathrm{~Hz}, 2 \mathrm{H}\right.$, $\mathrm{ArH}), 7.50\left(\mathrm{td}, J_{1}=7.8 \mathrm{~Hz}, J_{2}=1.2 \mathrm{~Hz}, 1 \mathrm{H}, \mathrm{ArH}\right), 7.80\left(\mathrm{dd}, J_{1}=8.0 \mathrm{~Hz}\right.$, $\left.J_{2}=0.8 \mathrm{~Hz}, 2 \mathrm{H}, \mathrm{ArH}\right)$.

${ }^{13} \mathrm{C} \mathrm{NMR}\left(\mathrm{CDCl}_{3}, 100 \mathrm{MHz}\right): \delta=44.08,126.93,127.58,127.87,128.55$, $128.74,131.52,134.30,138.14,167.37$.

\section{N-(Prop-2-yn-1-yl)benzamide (3r) ${ }^{24}$}

Eluent: PE/EtOAc (85:15); colorless oil; yield: $42.7 \mathrm{mg}$ (97\%).

${ }^{1} \mathrm{H}$ NMR $\left(\mathrm{CDCl}_{3}, 400 \mathrm{MHz}\right): \delta=2.22(\mathrm{t}, J=2.4 \mathrm{~Hz}, 1 \mathrm{H}, \mathrm{CH}), 4.18(\mathrm{dd}$, $\left.J_{1}=5.2 \mathrm{~Hz}, J_{2}=2.4 \mathrm{~Hz}, 2 \mathrm{H}, \mathrm{CH}_{2}\right), 7.01(\mathrm{~s}, 1 \mathrm{H}, \mathrm{NH}), 7.35\left(\mathrm{td}, J_{1}=7.6 \mathrm{~Hz}\right.$, $\left.J_{2}=0.4 \mathrm{~Hz}, 2 \mathrm{H}, \mathrm{ArH}\right), 7.44\left(\mathrm{td}, J_{1}=7.0 \mathrm{~Hz}, J_{2}=1.2 \mathrm{~Hz}, 1 \mathrm{H}, \mathrm{ArH}\right), 7.78(\mathrm{~d}$, $J=7.2 \mathrm{~Hz}, 2 \mathrm{H}, \mathrm{ArH})$.

${ }^{13} \mathrm{C} \mathrm{NMR}\left(\mathrm{CDCl}_{3}, 100 \mathrm{MHz}\right): \delta=29.66,71.54,79.66,127.16,128.48$, $131.69,133.67,167.44$

\section{$\mathbf{N}$-Cyclohexylbenzamide (3s) ${ }^{25}$}

Eluent: PE/EtOAc (80:20); white solid; yield: $54.2 \mathrm{mg}$ (98\%); mp 153$155^{\circ} \mathrm{C}$.

${ }^{1} \mathrm{H}$ NMR $\left(\mathrm{CDCl}_{3}, 400 \mathrm{MHz}\right): \delta=1.13-1.26\left(\mathrm{~m}, 3 \mathrm{H}, \mathrm{CH}_{2}\right), 1.34-1.44(\mathrm{~m}$, $\left.2 \mathrm{H}, \mathrm{CH}_{2}\right), 1.63$ (d, J = 12.8 Hz, $\left.1 \mathrm{H}, \mathrm{CH}\right), 1.71-1.74\left(\mathrm{~m}, 2 \mathrm{H}, \mathrm{CH}_{2}\right), 1.99$ $\left(\mathrm{d}, J=11.6 \mathrm{~Hz}, 2 \mathrm{H}, \mathrm{CH}_{2}\right), 3.91-3.98(\mathrm{~m}, 1 \mathrm{H}, \mathrm{CH}), 6.06(\mathrm{~s}, 1 \mathrm{H}, \mathrm{NH})$, 7.36-7.74 (m, 2 H, ArH), 7.43-7.47 (m, $1 \mathrm{H}, \mathrm{ArH}), 7.73$ (d, J = 7.2 Hz, 2 $\mathrm{H}, \mathrm{ArH})$.

${ }^{13} \mathrm{C} \mathrm{NMR}\left(\mathrm{CDCl}_{3}, 100 \mathrm{MHz}\right): \delta=24.91,25.49,33.10,48.67,126.85$, $128.39,131.12,135.02,166.65$.

\section{$\mathbf{N}$-[(3s,5s,7s)-Adamantan-1-yl]benzamide (3t) $)^{7 \mathrm{a}}$}

Eluent: PE/EtOAc (80:20); white solid; yield: $65.7 \mathrm{mg}(94 \%)$; mp 125$127^{\circ} \mathrm{C}$.

${ }^{1} \mathrm{H} \mathrm{NMR}\left(\mathrm{CDCl}_{3}, 400 \mathrm{MHz}\right): \delta=1.68(\mathrm{~s}, 6 \mathrm{H}), 2.09(\mathrm{~m}, 9 \mathrm{H}), 5.87(\mathrm{~s}, 1 \mathrm{H}$, $\mathrm{NH}), 7.34$ (t, $J=7.6 \mathrm{~Hz}, 2 \mathrm{H}, \mathrm{ArH}), 7.41$ (t, $J=7.2 \mathrm{~Hz}, 1 \mathrm{H}, \mathrm{ArH}), 7.68$ (d, $J=7.2 \mathrm{~Hz}, 2 \mathrm{H}, \mathrm{ArH})$.

${ }^{13} \mathrm{C} \mathrm{NMR}\left(\mathrm{CDCl}_{3}, 100 \mathrm{MHz}\right): \delta=29.44,36.32,41.57,52.20,126.68$, $128.35,130.94,135.95,166.60$.

\section{$N$-Dodecylbenzamide $(3 \mathrm{u})^{8 \mathrm{~d}}$}

Eluent: PE/EtOAc (90:10); white solid; yield: $73.4 \mathrm{mg}$ (97\%); mp 83$85^{\circ} \mathrm{C}$.

${ }^{1} \mathrm{H} \mathrm{NMR}\left(\mathrm{CDCl}_{3}, 400 \mathrm{MHz}\right): \delta=0.83\left(\mathrm{t}, J=6.8 \mathrm{~Hz}, 3 \mathrm{H}, \mathrm{CH}_{3}\right), 1.21-1.36$ $\left(\mathrm{m}, 16 \mathrm{H}, \mathrm{CH}_{2}\right), 1.55-1.58\left(\mathrm{~m}, 2 \mathrm{H}, \mathrm{CH}_{2}\right), 3.03(\mathrm{q}, J=7.6 ; 7.2 \mathrm{~Hz}, 2 \mathrm{H}$, $\left.\mathrm{CH}_{2}\right), 3.39\left(\mathrm{q}, J=6.8 ; 6.4 \mathrm{~Hz}, 2 \mathrm{H}, \mathrm{CH}_{2}\right), 6.38(\mathrm{~s}, 1 \mathrm{H}, \mathrm{NH}), 7.37(\mathrm{t}, J=6.8$ $\mathrm{Hz}, 2 \mathrm{H}, \mathrm{ArH}), 7.43(\mathrm{~d}, J=7.2 \mathrm{~Hz}, 1 \mathrm{H}, \mathrm{ArH}), 7.73(\mathrm{~d}, J=7.2 \mathrm{~Hz}, 2 \mathrm{H}$, ArH).

${ }^{13} \mathrm{C}$ NMR $\left(\mathrm{CDCl}_{3}, 100 \mathrm{MHz}\right): \mathrm{d}=08.55,14.06,22.62,26.96,29.28$, $29.50,29.53,29.58,31.85,40.06,45.74,126.83,128.42,131.19$, $134.80,167.49$.

\section{$N$-Dodecylacetamide $(3 v)^{26}$}

Eluent: PE/EtOAc (90:10); white solid; yield: $71.2 \mathrm{mg}$ (95\%); mp 52$54{ }^{\circ} \mathrm{C}$.

${ }^{1} \mathrm{H} \mathrm{NMR}\left(\mathrm{CDCl}_{3}, 400 \mathrm{MHz}\right): \delta=0.84\left(\mathrm{t}, J=6 \mathrm{~Hz}, 3 \mathrm{H}, \mathrm{CH}_{3}\right), 1.22-1.25$ $\left(\mathrm{m}, 18 \mathrm{H}, \mathrm{CH}_{2}\right), 1.45\left(\mathrm{t}, J=6.8 \mathrm{~Hz}, 2 \mathrm{H}, \mathrm{CH}_{2}\right), 1.94\left(\mathrm{~s}, 3 \mathrm{H}, \mathrm{CH}_{3}\right), 3.19(\mathrm{q}$, $\left.J=6.8 ; 6.4 \mathrm{~Hz}, 2 \mathrm{H}, \mathrm{CH}_{2}\right), 5.64(\mathrm{~s}, 1 \mathrm{H}, \mathrm{NH})$.

${ }^{13} \mathrm{C}$ NMR $\left(\mathrm{CDCl}_{3}, 100 \mathrm{MHz}\right): \delta=14.06,22.62,23.27,26.87,29.25$, $29.28,29.49,29.53,29.56,29.58,31.85,39.65,170.03$.

\section{(4-Methoxyphenyl)(piperidin-1-yl)methanone (5a) ${ }^{20}$}

Eluent: PE/EtOAc (80:20); white solid; yield: $66.9 \mathrm{mg}$ (96\%); mp 36$38^{\circ} \mathrm{C}$.

${ }^{1} \mathrm{H} \mathrm{NMR}\left(\mathrm{CDCl}_{3}, 400 \mathrm{MHz}\right): \delta=1.51-1.59\left(\mathrm{~m}, 6 \mathrm{H}, \mathrm{CH}_{2}\right), 3.36-3.56(\mathrm{~m}$, $\left.4 \mathrm{H}, \mathrm{CH}_{2}\right), 3.75\left(\mathrm{~s}, 3 \mathrm{H}, \mathrm{OCH}_{3}\right), 6.82(\mathrm{~d}, J=8.4 \mathrm{~Hz}, 2 \mathrm{H}, \mathrm{ArH}), 7.29$ (d, $J=8.8 \mathrm{~Hz}, 2 \mathrm{H}, \mathrm{ArH})$.

${ }^{13} \mathrm{C} \mathrm{NMR}\left(\mathrm{CDCl}_{3}, 100 \mathrm{MHz}\right): \delta=24.59,55.27,113.55,128.52,128.80$, $160.43,170.25$.

\section{(4-Nitrophenyl)(piperidin-1-yl)methanone (5b) ${ }^{20}$}

Eluent: PE/EtOAc (80:20); light yellow solid; yield: $62.4 \mathrm{mg}$ (89\%); mp $121-123^{\circ} \mathrm{C}$

${ }^{1} \mathrm{H}$ NMR $\left(\mathrm{CDCl}_{3}, 400 \mathrm{MHz}\right): \delta=1.43\left(\mathrm{~s}, 2 \mathrm{H}, \mathrm{CH}_{2}\right), 1.60\left(\mathrm{~s}, 4 \mathrm{H}, \mathrm{CH}_{2}\right)$, $3.19\left(\mathrm{~s}, 2 \mathrm{H}, \mathrm{CH}_{2}\right), 3.63\left(\mathrm{~s}, 2 \mathrm{H}, \mathrm{CH}_{2}\right), 7.46(\mathrm{~d}, J=6.8 \mathrm{~Hz}, 2 \mathrm{H}, \mathrm{ArH}), 8.15-$ $8.18(\mathrm{~m}, 2 \mathrm{H}, \mathrm{ArH})$. 
${ }^{13} \mathrm{C}$ NMR $\left(\mathrm{CDCl}_{3}, 100 \mathrm{MHz}\right): \delta=24.32,25.43,26.43,43.11,48.58$, $123.78,127.74,142.67,148.07,167.79$.

\section{$\boldsymbol{N}$-Isopropylbenzamide $(\mathbf{5 c})^{8 \mathrm{a}}$}

Eluent: PE/EtOAc (85:15); white solid; yield: 53.0 mg (94\%); mp 97$99{ }^{\circ} \mathrm{C}$.

${ }^{1} \mathrm{H} \mathrm{NMR}\left(\mathrm{CDCl}_{3}, 400 \mathrm{MHz}\right): \delta=1.23\left(\mathrm{~s}, 3 \mathrm{H}, \mathrm{CH}_{3}\right), 1.25\left(\mathrm{~s}, 3 \mathrm{H}, \mathrm{CH}_{3}\right)$, 4.23-4.31 (m, $1 \mathrm{H}, \mathrm{CH}), 6.01(\mathrm{~s}, 1 \mathrm{H}, \mathrm{NH}), 7.39(\mathrm{t}, J=8.0 \mathrm{~Hz}, 2 \mathrm{H}, \mathrm{ArH})$, 7.46 (t, J = 7.6 Hz, $1 \mathrm{H}, \mathrm{ArH}), 7.73$ (d, J = 7.2 Hz, $2 \mathrm{H}, \mathrm{ArH})$.

${ }^{13} \mathrm{C} \mathrm{NMR}\left(\mathrm{CDCl}_{3}, 100 \mathrm{MHz}\right): \delta=22.82,41.84,126.76,128.45,131.21$, 134.92, 166.65 .

\section{$\boldsymbol{N}$-Butylbenzamide (5d) $)^{8 \mathrm{~d}}$}

Eluent: PE/EtOAc (90:10); colorless oil; yield: $60.1 \mathrm{mg}$ (98\%).

${ }^{1} \mathrm{H} \mathrm{NMR}\left(\mathrm{CDCl}_{3}, 400 \mathrm{MHz}\right): \delta=0.92\left(\mathrm{~s}, 3 \mathrm{H}, \mathrm{CH}_{3}\right), 1.31-1.42(\mathrm{~m}, 2 \mathrm{H}$, $\left.\mathrm{CH}_{2}\right), 1.53-1.60\left(\mathrm{~m}, 2 \mathrm{H}, \mathrm{CH}_{2}\right), 3.39-3.44$ (q, $\mathrm{J}=7.2 ; 6.8 \mathrm{~Hz}, 2 \mathrm{H}, \mathrm{CH}_{2}$ ), $6.32(\mathrm{~s}, 1 \mathrm{H}, \mathrm{NH}), 7.38\left(\mathrm{td}, J_{1}=7.2 \mathrm{~Hz}, J_{2}=1.2 \mathrm{~Hz}, 2 \mathrm{H}, \mathrm{ArH}\right), 7.45$ (td, $\left.J_{1}=6.8 \mathrm{~Hz}, J_{2}=0.8 \mathrm{~Hz}, 1 \mathrm{H}, \mathrm{ArH}\right), 7.74\left(\mathrm{dd}, J_{1}=8.0 \mathrm{~Hz}, J_{2}=1.6 \mathrm{~Hz}, 2 \mathrm{H}\right.$, $\mathrm{ArH})$.

${ }^{13} \mathrm{C}$ NMR $\left(\mathrm{CDCl}_{3}, 100 \mathrm{MHz}\right): \delta=13.75,20.11,31.68,39.77,126.81$, 128.46, 131.23, 134.79, 167.55.

\section{$\mathrm{N}$-(tert-Butyl)benzamide (5e) $)^{27}$}

Eluent: PE/EtOAc (90:10); white solid; yield: $48.4 \mathrm{mg}$ (79\%); mp 131$133^{\circ} \mathrm{C}$.

${ }^{1} \mathrm{H} \mathrm{NMR}\left(\mathrm{CDCl}_{3}, 400 \mathrm{MHz}\right): \delta=1.23\left(\mathrm{~s}, 9 \mathrm{H}, 3 \times \mathrm{CH}_{3}\right), 5.96(\mathrm{~s}, 1 \mathrm{H}, \mathrm{NH})$, $7.31(\mathrm{t}, J=7.6 \mathrm{~Hz}, 2 \mathrm{H}, \mathrm{ArH}), 7.38(\mathrm{t}, J=7.2 \mathrm{~Hz}, 1 \mathrm{H}, \mathrm{ArH}), 7.64$ (dd, $\left.J_{1}=7.2 \mathrm{~Hz}, J_{2}=1.6 \mathrm{~Hz}, 2 \mathrm{H}, \mathrm{ArH}\right)$.

${ }^{13} \mathrm{C}$ NMR $\left(\mathrm{CDCl}_{3}, 100 \mathrm{MHz}\right): \delta=22.82,41.84,126.76,128.45,131.21$, $134.92,166.65$.

\section{$\mathbf{N}$-Benzyl-4-methoxybenzamide (7a) $)^{8 \mathrm{~d}}$}

Eluent: PE/EtOAc (80:20); white solid; yield: $86.4 \mathrm{mg}$ (95\%); mp 122$124^{\circ} \mathrm{C}$.

${ }^{1} \mathrm{H} \mathrm{NMR}\left(\mathrm{CDCl}_{3}, 400 \mathrm{MHz}\right): \delta=3.80\left(\mathrm{~s}, 3 \mathrm{H}, \mathrm{OCH}_{3}\right), 4.56(\mathrm{~d}, J=6 \mathrm{~Hz}, 2$ $\left.\mathrm{H}, \mathrm{CH}_{2}\right), 6.75$ (br, $\left.1 \mathrm{H}, \mathrm{NH}\right), 6.84-6.87(\mathrm{~m}, 2 \mathrm{H}, \mathrm{ArH}), 7.23-7.30(\mathrm{~m}, 5 \mathrm{H}$, ArH), $7.75(\mathrm{~d}, J=8.4 \mathrm{~Hz}, 2 \mathrm{H}, \mathrm{ArH})$.

$\left.{ }^{13} \mathrm{C} \mathrm{NMR} \mathrm{(CDCl}, 100 \mathrm{MHz}\right): \delta=43.91,55.34,113.65,126.59,127.38$, $127.78,128.64,128.83,138.49,162.12,166.96$.

\section{$\boldsymbol{N}$-Benzyl-4-(trifluoromethyl)benzamide (7b) $)^{8 \mathrm{~d}}$}

Eluent: PE/EtOAc (80:20); white solid; yield: 86.6 mg (94\%); mp 149$151^{\circ} \mathrm{C}$.

${ }^{1} \mathrm{H} \mathrm{NMR}\left(\mathrm{CDCl}_{3}, 400 \mathrm{MHz}\right): \delta=4.64\left(\mathrm{~d}, J=5.6 \mathrm{~Hz}, 2 \mathrm{H}, \mathrm{CH}_{2}\right), 6.61(\mathrm{br}, 1$ $\mathrm{H}, \mathrm{NH}$ ), 7.29-7.38 (m, $5 \mathrm{H}, \mathrm{ArH}$ ), 7.67 (d, J = 8.4 Hz, $2 \mathrm{H}, \mathrm{ArH}$ ), 7.88 (d, $J=8.0 \mathrm{~Hz}, 2 \mathrm{H}, \mathrm{ArH})$.

${ }^{13} \mathrm{C} \mathrm{NMR}\left(\mathrm{CDCl}_{3}, 100 \mathrm{MHz}\right): \delta=44.77,122.74,125.45,126.11(\mathrm{~J}=3.6$ $\mathrm{Hz}), 128.29,134.78(\mathrm{~J}=33.8 \mathrm{~Hz}), 138.09,138.9,138.19,166.56$.

\section{$\mathbf{N}$-Cyclohexyl-4-(trifluoromethyl)benzamide (7c) ${ }^{28}$}

Eluent: PE/EtOAc (75:25); white solid; $85.0 \mathrm{~g}(95 \%) ; \mathrm{mp} 167-169{ }^{\circ} \mathrm{C}$. ${ }^{1} \mathrm{H}$ NMR $\left(\mathrm{CDCl}_{3}, 400 \mathrm{MHz}\right): \delta=1.16-1.28\left(\mathrm{~m}, 3 \mathrm{H}, \mathrm{CH}_{2}\right), 1.36-1.46(\mathrm{~m}$, $\left.2 \mathrm{H}, \mathrm{CH}_{2}\right), 1.63-1.67(\mathrm{~m}, 1 \mathrm{H}, \mathrm{CH}), 1.75\left(\mathrm{dt}, J_{1}=13.6 \mathrm{~Hz}, J_{2}=3.6 \mathrm{~Hz}, 2 \mathrm{H}\right.$, $\left.\mathrm{CH}_{2}\right), 2.02\left(\mathrm{dd}, J_{1}=12.4 \mathrm{~Hz}, J_{2}=3.2 \mathrm{~Hz}, 2 \mathrm{H}, \mathrm{CH}_{2}\right), 3.91-4.01(\mathrm{~m}, 1 \mathrm{H}$, $\mathrm{CH}), 6.03$ (br, $1 \mathrm{H}, \mathrm{NH}) 7.66$ (d, J = 8.0 Hz, $2 \mathrm{H}, \mathrm{ArH}), 7.83(\mathrm{~d}, J=8.4 \mathrm{~Hz}$, $2 \mathrm{H}, \mathrm{ArH})$.
${ }^{13} \mathrm{C}$ NMR $\left(\mathrm{CDCl}_{3}, 100 \mathrm{MHz}\right): \delta=25.35,25.97,33.62,49.47,122.79$, 125.51, $126.042(J=3.4 \mathrm{~Hz}), 127.79,133.47(J=32.5 \mathrm{~Hz}), 138.84$, 165.83.

\section{Piperidin-1-yl[4-(trifluoromethyl)phenyl]methanone (7d) ${ }^{20}$}

Eluent: PE/EtOAc (85:15); white solid; yield: 84.0 g (99\%); mp 98$100{ }^{\circ} \mathrm{C}$.

${ }^{1} \mathrm{H} \mathrm{NMR}\left(\mathrm{CDCl}_{3}, 400 \mathrm{MHz}\right): \delta=1.43\left(\mathrm{~s}, 2 \mathrm{H}, \mathrm{CH}_{2}\right), 1.60\left(\mathrm{~s}, 4 \mathrm{H}, \mathrm{CH}_{2}\right)$, $3.32\left(\mathrm{~s}, 2 \mathrm{H}, \mathrm{CH}_{2}\right), 3.64\left(\mathrm{~s}, 2 \mathrm{H}, \mathrm{CH}_{2}\right), 7.42(\mathrm{~d}, J=8.0 \mathrm{~Hz}, 2 \mathrm{H}, \mathrm{ArH}), 7.58$ (d, $J=8.0 \mathrm{~Hz}, 2 \mathrm{H}, \mathrm{ArH}$ ).

${ }^{13} \mathrm{C}$ NMR $\left(\mathrm{CDCl}_{3}, 100 \mathrm{MHz}\right): \delta=24.88,25.97,26.94,43.56,49.09$, $122.88,125.58,125.95(\mathrm{~J}=3.7 \mathrm{~Hz}), 127.58,13.72(\mathrm{~J}=32.6 \mathrm{~Hz}), 140.55$, 169.20 .

\section{Morpholino[4-(trifluoromethyl)phenyl]methanone (7e $)^{7 \mathrm{a}}$}

Eluent: PE/EtOAc (80:20); white solid; yield: $81.2 \mathrm{mg}$ (97\%); mp 47$49^{\circ} \mathrm{C}$.

${ }^{1} \mathrm{H}$ NMR $\left(\mathrm{CDCl}_{3}, 400 \mathrm{MHz}\right): \delta=3.32\left(\mathrm{~s}, 2 \mathrm{H}, \mathrm{CH}_{2}\right), 3.54\left(\mathrm{~s}, 2 \mathrm{H}, \mathrm{CH}_{2}\right)$, $3.71\left(\mathrm{~s}, 4 \mathrm{H}, \mathrm{CH}_{2}\right), 7.46$ (d, J = 7.6 Hz, $\left.2 \mathrm{H}, \mathrm{ArH}\right), 7.61$ (d, J = 8.0 Hz, $2 \mathrm{H}$, ArH).

${ }^{13} \mathrm{C}$ NMR $\left(\mathrm{CDCl}_{3}, 100 \mathrm{MHz}\right): \delta=42.42,47.99,66.68,119.53,122.24$, $124.95,125.60(J=3.5 \mathrm{~Hz}), 127.40,131.68(J=31.6 \mathrm{~Hz}), 138.82$, 168.81 .

\section{Pyrrolidin-1-yl[4-(trifluoromethyl)phenyl]methanone (7f $)^{24}$}

Eluent: PE/EtOAc (85:15); white solid; yield: $77.8 \mathrm{mg}$ (97\%); mp 80$82^{\circ} \mathrm{C}$.

${ }^{1} \mathrm{H}$ NMR $\left(\mathrm{CDCl}_{3}, 400 \mathrm{MHz}\right): \delta=1.84$ (pent, $\left.J=6.5 \mathrm{~Hz}, 2 \mathrm{H}, \mathrm{CH}_{2}\right), 1.93$ (pent, $\left.J=6.7 \mathrm{~Hz}, 2 \mathrm{H}, \mathrm{CH}_{2}\right), 3.34\left(\mathrm{t}, J=6.4 .0 \mathrm{~Hz}, 2 \mathrm{H}, \mathrm{CH}_{2}\right), 3.61(\mathrm{t}, J=6.8$ $\left.\mathrm{Hz}, 2 \mathrm{H}, \mathrm{CH}_{2}\right), 7.67-7.63$ ( $\left.\mathrm{m}, 4 \mathrm{H}, \mathrm{ArH}\right)$.

${ }^{13} \mathrm{C} \mathrm{NMR}\left(\mathrm{CDCl}_{3}, 100 \mathrm{MHz}\right): \delta=24.84,26.81,46.70,49.93,125.58$, $125.81(J=3.6 \mathrm{~Hz}), 127.90,132.08(J=32.7 \mathrm{~Hz}), 141.15,168.68$.

\section{Transamidation of Activated Amide 1a with Amino Acids and Ami- no Alcohols}

The amide substrate $\mathbf{1 a}(0.273 \mathrm{mmol})$ and the respective amino acid or amino alcohol $(0.356 \mathrm{mmol})$ in EtOH $(1 \mathrm{~mL})$ were taken in a vial and the reaction mixture was stirred at $60{ }^{\circ} \mathrm{C}(1-3 \mathrm{~h})$. The mixture was concentrated in vacuo and the crude product was purified by column chromatography as described in the general procedure to give the corresponding transamidation product $\mathbf{8 a}-\mathbf{d}$.

\section{Methyl Benzoyl-L-phenylalaninate (8a) $)^{29}$}

Eluent: PE/EtOAc (85:15); white solid; yield: $75.1 \mathrm{mg}$ (97\%); mp 84$86{ }^{\circ} \mathrm{C} ;[\alpha]_{\mathrm{D}}^{20}+65.56\left(\right.$ c $\left.0.1, \mathrm{CHCl}_{3}\right)$.

${ }^{1} \mathrm{H} \mathrm{NMR}\left(\mathrm{CDCl}_{3}, 400 \mathrm{MHz}\right): \delta=3.19-3.31\left(\mathrm{~m}, 2 \mathrm{H}, \mathrm{CH}_{2}\right), 3.75(\mathrm{~s}, 3 \mathrm{H}$, $\left.\mathrm{OCH}_{3}\right), 5.08$ (q, J = 5.6 Hz, $\left.1 \mathrm{H}, \mathrm{CH}\right), 6.57$ (d, J = 5.6 Hz, $\left.1 \mathrm{H}, \mathrm{NH}\right), 7.12$ (d, $J=7.2 \mathrm{~Hz}, 2 \mathrm{H}, \mathrm{ArH}), 7.24-7.30(\mathrm{~m}, 3 \mathrm{H}, \mathrm{ArH}), 7.41(\mathrm{t}, J=7.2 \mathrm{~Hz}, 2 \mathrm{H}$, ArH), 7.49 (t, J= 7.2 Hz, $1 \mathrm{H}, \mathrm{ArH}), 7.71(\mathrm{~d}, J=7.6 \mathrm{~Hz}, 2 \mathrm{H}, \mathrm{ArH})$.

${ }^{13} \mathrm{C}$ NMR $\left(\mathrm{CDCl}_{3}, 100 \mathrm{MHz}\right): \delta=37.80,53.40,53.56,127.02,127.15$, $128.56,128.59,129.29,131.76,133.78,135.88,166.93,172.10$.

\section{Methyl Benzoyl-L-methioninate (8b) $)^{30}$}

Eluent: PE/EtOAc (85:15); white solid; yield: $68.8 \mathrm{mg}$ (94\%); $\mathrm{mp} 87-$ $89^{\circ} \mathrm{C} ;[\alpha]_{\mathrm{D}}{ }^{20}+19.5\left(\right.$ c $\left.0.1, \mathrm{CHCl}_{3}\right)$.

${ }^{1} \mathrm{H} \mathrm{NMR}\left(\mathrm{CDCl}_{3}, 400 \mathrm{MHz}\right): \delta=2.08\left(\mathrm{~s}, 4 \mathrm{H}, \mathrm{CH}_{2}\right), 2.26(\mathrm{~s}, 1 \mathrm{H}, \mathrm{CH}), 2.56$ $\left(\mathrm{s}, 2 \mathrm{H}, \mathrm{CH}_{2}\right), 3.76\left(\mathrm{~s}, 3 \mathrm{H}, \mathrm{OCH}_{3}\right), 4.906(\mathrm{~s}, 1 \mathrm{H}, \mathrm{CH}), 6.99(\mathrm{~s}, 1 \mathrm{H}, \mathrm{NH})$, 7.24-7.48 (m, $3 \mathrm{H}, \mathrm{ArH}), 7.79$ (s, $2 \mathrm{H}, \mathrm{ArH})$. 
${ }^{13} \mathrm{C}$ NMR $\left(\mathrm{CDCl}_{3}, 100 \mathrm{MHz}\right): \delta=15.47,30.03,31.49,52.01,52.60$, $127.05,128.57,131.81,133.64,167.05,172.55$.

\section{Ethyl Benzoyl-L-alaninate (8c) ${ }^{31}$}

Eluent: PE/EtOAc (85:15); white solid; yield: $53.3 \mathrm{mg}$ (94\%); mp 76$78{ }^{\circ} \mathrm{C} ;[\alpha]_{\mathrm{D}}^{20}+30.14\left(\mathrm{c} 0.1, \mathrm{CHCl}_{3}\right)$.

${ }^{1} \mathrm{H} \mathrm{NMR}\left(\mathrm{CDCl}_{3}, 400 \mathrm{MHz}\right): \delta=1.29\left(\mathrm{t}, J=7.2 \mathrm{~Hz}, 3 \mathrm{H}, \mathrm{CH}_{2}\right), 1.50(\mathrm{~d}$, $\left.J=7.2 \mathrm{~Hz}, 3 \mathrm{H}, \mathrm{CH}_{3}\right), 5.72\left(\mathrm{q}, J=7.2 \mathrm{~Hz}, 2 \mathrm{H}, \mathrm{CH}_{2}\right), 4.76$ (pent, $J=7.2 \mathrm{~Hz}$, $1 \mathrm{H}, \mathrm{CH}), 6.79(\mathrm{~s}, 1 \mathrm{H}, \mathrm{NH}), 7.41(\mathrm{t}, J=7.2 \mathrm{~Hz}, 2 \mathrm{H}, \mathrm{ArH}), 7.49(\mathrm{~d}, J=7.2$ $\mathrm{Hz}, 1 \mathrm{H}, \mathrm{ArH}), 7.79$ (d, J = 7.2 Hz, $2 \mathrm{H}, \mathrm{ArH})$.

${ }^{13} \mathrm{C}$ NMR $\left(\mathrm{CDCl}_{3}, 100 \mathrm{MHz}\right): \delta=14.10,18.66,48.51,61.62,126.98$, $128.53,131.66,133.91,166.77,173.25$.

\section{$\mathbf{N}$-[(2R,3R)-1-Hydroxy-3-methylpentan-2-yl]benzamide (8d)}

Eluent: PE/EtOAc (80:20); white solid; yield: $53.9 \mathrm{mg}$ (89\%); mp 79$81{ }^{\circ} \mathrm{C} ;[\alpha]_{D}{ }^{20}-8.28\left(\right.$ c $\left.0.1, \mathrm{CHCl}_{3}\right)$.

${ }^{1} \mathrm{H}$ NMR $\left(\mathrm{CDCl}_{3}, 400 \mathrm{MHz}\right): \delta=0.91\left(\mathrm{t}, J=7.2 \mathrm{~Hz}, 3 \mathrm{H}, \mathrm{CH}_{3}\right), 0.97(\mathrm{~d}$, $\left.J=6.8 \mathrm{~Hz}, 3 \mathrm{H}, \mathrm{CH}_{3}\right), 1.16-1.27\left(\mathrm{~m}, 2 \mathrm{H}, \mathrm{CH}_{2}\right), 1.53-1.61(\mathrm{~m}, 1 \mathrm{H}, \mathrm{CH})$, 1.75-1.76 (m, $1 \mathrm{H}, \mathrm{CH}), 3.73-3.80\left(\mathrm{~m}, 2 \mathrm{H}, \mathrm{CH}_{2}\right), 3.99(\mathrm{t}, J=7.2 \mathrm{~Hz}, 1 \mathrm{H}$, $\mathrm{OH}), 6.48(\mathrm{~d}, J=7.2 \mathrm{~Hz}, 1 \mathrm{H}, \mathrm{NH}), 7.39(\mathrm{t}, J=7.6 \mathrm{~Hz}, 2 \mathrm{H}, \mathrm{ArH}), 7.47(\mathrm{t}$, $J=7.2 \mathrm{~Hz}, 1 \mathrm{H}, \mathrm{ArH}), 7.74(\mathrm{~d}, J=7.2 \mathrm{~Hz}, 2 \mathrm{H}, \mathrm{ArH})$.

${ }^{13} \mathrm{C}$ NMR $\left(\mathrm{CDCl}_{3}, 100 \mathrm{MHz}\right): \delta=11.34,15.60,25.63,35.73,56.25$, $63.53,126.93,128.54,131.54,134.41,168.34$.

HRMS: $\mathrm{m} / \mathrm{z}$ calcd for $\mathrm{C}_{13} \mathrm{H}_{19} \mathrm{NO}_{2} \mathrm{Na}[\mathrm{M}+\mathrm{Na}]^{+}$: 244.1313; found: 244.1318 .

\section{Transamidation of Activated Secondary Amides in Water; General Procedure}

The amide substrate $\mathbf{1}$ or $\mathbf{6 b}$ (1 equiv) and the respective amine (4 equiv) in $\mathrm{H}_{2} \mathrm{O}(1 \mathrm{~mL})$ were taken in a vial and the reaction mixture was stirred at $60{ }^{\circ} \mathrm{C}(1-2 \mathrm{~h})$. After the reaction was complete, EtOAC $(20 \mathrm{~mL})$ was added. The organic layer was separated, dried (anhyd $\mathrm{Na}_{2} \mathrm{SO}_{4}$ ), and the filtrate was concentrated. The crude product was purified by column chromatography on silica gel to afford the corresponding transamidation product.

\section{Furan-2-yl(morpholino)methanone (9) ${ }^{32}$}

According to the general procedure, the reaction of $\mathbf{1 f}(0.28 \mathrm{mmol})$ and morpholine $(1.13 \mathrm{mmol})$ in $\mathrm{H}_{2} \mathrm{O}(1 \mathrm{~mL})$ at $60{ }^{\circ} \mathrm{C}$ for $2 \mathrm{~h}$, afforded the corresponding transamidation product $9(46.9 \mathrm{mg}, 92 \%)$ after column chromatography (PE/EtOAc, 85:15); white solid; mp 51-53 ${ }^{\circ} \mathrm{C}$.

${ }^{1} \mathrm{H}$ NMR $\left(\mathrm{CDCl}_{3}, 400 \mathrm{MHz}\right): \delta=3.69-3.77\left(\mathrm{~m}, 8 \mathrm{H}, \mathrm{CH}_{2}\right), 6.44-6.45(\mathrm{~m}$, $1 \mathrm{H}, \mathrm{ArH}), 6.98(\mathrm{~d}, J=3.6 \mathrm{~Hz}, 1 \mathrm{H}, \mathrm{ArH}), 7.43-7.44(\mathrm{~m}, 1 \mathrm{H}, \mathrm{ArH})$.

${ }^{13} \mathrm{C} \mathrm{NMR}\left(\mathrm{CDCl}_{3}, 100 \mathrm{MHz}\right): \delta=66.89,111.32,116.73,143.73,147.66$, 159.04 .

\section{Comparative Studies}

The amide substrate $\mathbf{1 0}$ or $\mathbf{1 1}$ (1.0 equiv) and the respective amine (1.3 equiv) in ethanol $(1 \mathrm{~mL})$ were taken in a vial and the reaction mixture was stirred at $45-60{ }^{\circ} \mathrm{C}(1-12 \mathrm{~h})$. After completion of the reaction, the mixture was concentrated. The crude product was purified by column chromatography (PE/EtOAc) on $\mathrm{SiO}_{2}$ to give the corresponding transamidation product.

\section{$\mathbf{N}$-(2,6-Dimethylphenyl)benzamide (12a) $)^{7 a}$}

Eluent: PE/EtOAc (85:15); white solid; yield: 49.9 mg (69\%); mp 155$157^{\circ} \mathrm{C}$.
${ }^{1} \mathrm{H} \mathrm{NMR}\left(\mathrm{CDCl}_{3}, 400 \mathrm{MHz}\right): \delta=2.27\left(\mathrm{~s}, 6 \mathrm{H}, \mathrm{CH}_{3}\right), 7.12(\mathrm{~m}, 3 \mathrm{H}, \mathrm{ArH})$, $7.38(\mathrm{~s}, 1 \mathrm{H}, \mathrm{ArH}), 7.49$ (t, $J=7.6 \mathrm{~Hz}, 1 \mathrm{H}, \mathrm{ArH}), 7.56(\mathrm{t}, J=7.2 \mathrm{~Hz}, 1 \mathrm{H}$, $\operatorname{ArH}), 7.91(\mathrm{~d}, J=7.2 \mathrm{~Hz}, 1 \mathrm{H}, \mathrm{ArH})$.

${ }^{13} \mathrm{C} \mathrm{NMR}\left(\mathrm{CDCl}_{3}, 100 \mathrm{MHz}\right): \delta=18.42,127.21,127.34,128.21,128.67$, $131.69,133.93,134.43,135.58,165.91$.

\section{$\boldsymbol{N}$-Benzyl-4-bromobenzamide $(\mathbf{1 2 b})^{8 \mathrm{~d}}$}

Eluent: PE/EtOAc (80:20); white solid; yield: $71.4 \mathrm{mg}$ (96\%); mp 125$127^{\circ} \mathrm{C}$.

${ }^{1} \mathrm{H} \mathrm{NMR}\left(\mathrm{CDCl}_{3}, 400 \mathrm{MHz}\right): \delta=4.62\left(\mathrm{~d}, J=5.2 \mathrm{~Hz}, 2 \mathrm{H}, \mathrm{CH}_{2}\right), 6.62(\mathrm{~s}, 1$ H, NH), 7.28-7.35 (m, 5 H, ArH), 7.55 (d, J = 8.0 Hz, 2 H, ArH), 7.66 (d, $J=8.4 \mathrm{~Hz}, 2 \mathrm{H}, \mathrm{ArH})$.

${ }^{13} \mathrm{C}$ NMR $\left(\mathrm{CDCl}_{3}, 100 \mathrm{MHz}\right): \delta=44.68,126.71,128.19,128.39,129.08$, $129.30,132.28,133.64,138.38,166.91$.

\section{(S)-3,3-Diphenyl- $N$-(1-phenylethyl)propanamide (12c) $)^{11}$}

Eluent: PE/EtOAc (90:10); yellowish solid; yield: $64.2 \mathrm{mg}$ (88\%); mp $121-123{ }^{\circ} \mathrm{C} ;[\alpha]_{\mathrm{D}}^{20}+46.18\left(\right.$ c $\left.0.1, \mathrm{CHCl}_{3}\right)$.

${ }^{1} \mathrm{H}$ NMR ( $\left.\mathrm{CDCl}_{3}, 400 \mathrm{MHz}\right): \delta=1.25\left(\mathrm{~d}, J=7.2 \mathrm{~Hz}, 3 \mathrm{H}, \mathrm{CH}_{3}\right), 2.90(\mathrm{~d}$, $J=8.0 \mathrm{~Hz}, 2 \mathrm{H}, \mathrm{CH}_{2}$ ), 4.56 (t, $J=7.6 \mathrm{~Hz}, 1 \mathrm{H}, \mathrm{CH}$ ), 4.98 (pent, $J=7.2 \mathrm{~Hz}$, $1 \mathrm{H}, \mathrm{CH}), 5.65(\mathrm{~s}, 1 \mathrm{H}, \mathrm{NH}), 6.98(\mathrm{~d}, J=7.6 \mathrm{~Hz}, 2 \mathrm{H}, \mathrm{ArH}), 7.21-7.31(\mathrm{~m}$, $12 \mathrm{H}, \mathrm{ArH})$.

${ }^{13} \mathrm{C}$ NMR $\left(\mathrm{CDCl}_{3}, 100 \mathrm{MHz}\right): \delta=21.31,43.51,47.62,48.37,125.97$, $126.52,127.08,127.77,128.47,128.60,142.80,143.48,143.66$, 170.14 .

\section{Funding Information}

R.R. thanks the University Grants Commission, India for a fellowship under Dr. D. S. Kothari Postdoctoral Scheme (No. CH/14-15/0132). SCN thanks Indian National Science Academy, New Delhi for the award of INSA Distinguished Professorship.

\section{Supporting Information}

Supporting information (copies of ${ }^{1} \mathrm{H}$ and ${ }^{13} \mathrm{NMR}$ spectra of all compounds) for this article is available online at https://doi.org/10.1055/s-0037-1610664.

\section{References}

(1) (a) Zabicky, J. The Chemistry of Amides; Wiley-VCH: New York, 1970. (b) Greenberg, A.; Breneman, C. M.; Liebman, J. F. The Amide Linkage: Structural Significance in Chemistry, Biochemistry and Materials Science; Wiley: New York, 2003.

(2) (a) Cheung, C. W.; Ploeger, M. L.; Hu, X. Nat. Commun. 2017, 8, 14878. (b) de Figueiredo, R. M.; Suppo, J.-S.; Campagne, J.-M. Chem. Rev. 2016, 116, 12029. (c) Hudlicky, T.; Reed, J. W. The Way of Synthesis: Evolution of Design and Methods for Natural Products; Wiley-VCH: Weinheim, 2007. (d) Corey, E. J.; Cheng, X.-M. The Logic of Chemical Synthesis; Wiley-VCH: Weinheim, 1995.

(3) (a) Pattabiraman, V. R.; Bode, J. W. Nature 2011, 480, 471. (b) Ojeda-Porras, A.; Gamba-Sanchez, D. J. Org. Chem. 2016, 81, 11548. (c) Aubé, J. Angew. Chem. Int. Ed. 2012, 51, 3063. (d) Allen, A. C.; Atkinson, B. N.; Williams, J. M. Angew. Chem. Int. Ed. 2012, 51, 1383. (e) Pauling, L.; Corey, R. B.; Branson, H. R. Proc. Natl. Acad. Sci. U. S. A. 1951, 37, 205. 
(4) (a) Dineen, T. A.; Zajac, M. A.; Myers, A. G. J. Am. Chem. Soc. 2006, 128, 16406. (b) Becerra-Figueroa, L.; Ojeda-Porras, A.; Gamba-Sánchez, D. J. J. Org. Chem. 2014, 79, 4544. (c) Rao, S. N.; Mohan, D. C.; Adimurthy, S. Green Chem. 2014, 16, 4122. (d) Jia, M.; Zhang, H.; Lin, Y.; Chen, D.; Chen, Y.; Xia, Y. Org. Biomol. Chem. 2018, 16, 3615. (e) Tani, H.; Oguni, N.; Araki, T. Bull. Chem. Soc.Jpn. 1964, 37, 1245. (f) Piazzolla, F.; Temperini, A. Tetrahedron Lett. 2018, 59, 2615.

(5) (a) Cheung, C. W.; Ma, J.-A.; Hu, X. J. Am. Chem. Soc. 2018, 140, 6789. (b) Nguyen, T. B.; Sorres, J.; Tran, M. Q.; Ermolenko, L.; AlMourabit, A. Org. Lett. 2012, 14, 3202. (c) Rao, S. N.; Mohan, D. C.; Adimurthy, S. Org. Lett. 2013, 15, 1496. (d) Stephenson, N. A.; Zhu, J.; Gellman, S. H.; Stahl, S. S. J. Am. Chem. Soc. 2009, 131, 10003.

(6) (a) Eldred, S. E.; Stone, D. A.; Gellman, S. H.; Stahl, S. S. J. Am. Chem. Soc. 2003, 125, 3422. (b) Bon, E.; Bigg, D. C. H.; Bertrand, G. J. Org. Chem. 1994, 59, 4035.

(7) (a) Baker, E. L.; Yamano, M. M.; Zhou, Y.; Anthony, S. M.; Garg, N. K. Nat. Commun. 2016, 7, 11554. (b) Dander, J. E.; Baker, E. L.; Garg, N. K. Chem. Sci. 2017, 8, 6433. (c) Boit, T. B.; Weires, N. A.; Kim, J.; Garg, N. K. ACS Catal. 2018, 8, 1003. (d) Medina, J. M.; Moreno, J.; Racine, S.; Du, S.; Garg, N. K. Angew. Chem. Int. Ed. 2017, 56, 6567.

(8) (a) Liu, Y.; Shi, S.; Achtenhagen, M.; Liu, R.; Szostak, M. Org. Lett. 2017, 19, 1614. (b) Liu, Y.; Achtenhagen, M.; Liu, R.; Szostak, M. Org. Biomol. Chem. 2018, 16, 1322. (c) Meng, G.; Lei, P.; Szostak, M. A. Org. Lett. 2017, 19, 2158. (d) Shi, S.; Szostak, M. Chem. Commum. 2017, 53, 10584. (e) Li, G.; Lei, P.; Szostak, M.; CasalsCruañas, E.; Poater, A.; Cavallo, L.; Nolan, S. P. ChemCatChem 2018, 10, 3096. (f) Meng, G.; Szostak, M. Eur. J. Org. Chem. 2018, 2352. (g) Bisz, E.; Piontek, A.; Dziuk, B.; Szostak, R.; Szostak, M. J. Org. Chem. 2018, 83, 3159. (h) Szostak, R.; Szostak, M. Org. Lett. 2018, 20, 1342.

(9) (a) Greenberg, A.; Venanzi, C. A. J. Am. Chem. Soc. 1993, 115, 6951. (b) Greenberg, A.; Moore, D. T.; DuBois, T. D. J. Am. Chem. Soc. 1996, 118, 8658. (c) Szostak, R.; Meng, G.; Szostak, M. J. Org. Chem. 2017, 82, 6373. (d) Kirby, A. J.; Komarov, I. V.; Wothers, P. D.; Feeder, N. Angew. Chem. Int. Ed. 1998, 37, 785. (e) Szostak, R.; Shi, S.; Meng, G.; Lalancette, R.; Szostak, M. J. Org. Chem. 2016, 81, 8091. (f) Pace, V.; Holzer, W.; Meng, G.; Shi, S.; Lalancette, R.; Szostak, R.; Szostak, M. Chem. Eur. J. 2016, 22, 14494. (g) Szostak, R.; Aubé, J.; Szostak, M. Chem. Commun. 2015, 51, 6395.
(10) (a) Hie, L.; Nathel, N. F. F.; Hong, X.; Yang, Y. F.; Houk, K. N.; Garg, N. K. Angew. Chem. Int. Ed. 2016, 55, 2810. (b) Pu, X.; Hu, J.; Zhao, Y.; Shi, Z. ACS Catal. 2016, 6, 6692. (c) Hie, L.; Baker, E. L.; Anthony, S. M.; Desrosiers, J. N.; Senanayake, C.; Garg, N. K. Angew. Chem. Int. Ed. 2016, 55, 15129.

(11) While our manuscript was in preparation, Verho reported a two-step procedure for the transamidation of 8-aminoquinoline amides proceeding via the intermediate $\mathrm{N}$-acyl-Boc-carbamates: Verho, O.; Lati, M. P.; Oschmann, M. A. J. Org. Chem. 2018, 83. 4464.

(12) Xuan, J.; Li, B.-J.; Feng, Z.-J.; Sun, G.-D.; Ma, H.-H.; Yuan, Z.-W.; Chen, J.-R.; Lu, L.-Q.; Xiao, W.-J. Chem. Asian J. 2013, 8, 1090.

(13) Xu, S.; Liu, J.; Hu, D.; Bi, X. Green Chem. 2015, 17, 184.

(14) Wang, C.; Huang, L.; Wang, F.; Zou, G. Tetrahedron Lett. 2018, 59, 2299.

(15) Ovian, J. M.; Kelly, C. B.; Pistritto, V. A.; Leadbeater, N. E. Org. Lett. 2017, 19, 1286.

(16) Maji, M.; Chakrabarti, K.; Paul, B.; Roy, B. C.; Kundu, S. Adv. Synth. Catal. 2018, 360, 722.

(17) Takahata, H.; Yamazaki, T. J. Org. Chem. 1985, 50, 4648.

(18) Qiu, F.; Yang, W.; Chang, Y.; Guan, B. Asian J. Org. Chem. 2017, 6, 1361.

(19) Braddock, D. C.; Lickiss, P. D.; Rowley, B. C.; Pugh, D.; Purnomo, T.; Santhakumar, G.; Fussell, S. J. Org. Lett. 2018, 20, 950.

(20) Kovalenko, O. O.; Volkov, A.; Adolfsson, H. Org. Lett. 2015, 17, 446.

(21) Lee, H. J.; Lee, J. I. J. Korean Chem. Soc. 2017, 61, 286.

(22) Yuan, Y. C.; Kamaraj, R.; Bruneau, C.; Labasque, T.; Roisnel, T.; Gramage, D. R. Org. Lett. 2017, 19, 6404.

(23) Wang, J.; Yin, X.; Wu, J.; Wu, D.; Pan, Y. Tetrahedron 2013, 69, 10463.

(24) Kumar, V.; Connon, S. J. Chem. Commun. 2017, 53, 10212.

(25) Vanos, C. M.; Lambert, T. H. Chem. Sci. 2010, 1, 705.

(26) Olivo, G.; Farinelli, G.; Barbieri, A.; Lanzalunga, O.; Di Stefano, S.; Costas, M. Angew. Chem. Int. Ed. 2017, 56, 16347.

(27) Kim, H.; Shin, K.; Chang, S. J. Am. Chem. Soc. 2014, 136, 5904.

(28) Prosser, A. R.; Banning, J. E.; Rubina, M.; Rubin, M. Org. Lett. 2010, 12, 3968.

(29) Metrano, A. J.; Miller, S. J. J. Org. Chem. 2014, 79, 1542.

(30) Mugherli, L.; Burchak, O. N.; Balakireva, L. A.; Thomas, A.; Chatelain, F.; Balakirev, M. Y. Angew. Chem. Int. Ed. 2009, 48, 7639.

(31) Karnik, A. V.; Kamath, S. S. J. Org. Chem. 2007, 72, 7435.

(32) Gu, J. J.; Fang, Z.; Liu, C. K.; Yang, Z.; Li, X.; Wei, P.; Guo, K. RSC Adv. 2015, 5, 95014. 